\title{
The composition of ices in comet C/1995 01 (Hale-Bopp) from radio spectroscopy
}

\section{Further results and upper limits on undetected species}

\author{
J. Crovisier ${ }^{1}$, D. Bockelée-Morvan ${ }^{1}$, P. Colom ${ }^{1}$, N. Biver ${ }^{1}$, D. Despois ${ }^{2}$, \\ D. C. Lis $^{3}$, and the Team for target-of-opportunity radio observations of comets
}

1 Observatoire de Paris, 92195 Meudon, France

2 Observatoire de Bordeaux, BP 89, 33270 Floirac, France

3 California Institute of Technology, MS 320-47, Pasadena, CA 91125, USA

Received 14 November 2003 / Accepted 26 January 2004

\begin{abstract}
From radio spectroscopic observations of comets, more than 22 molecules, radicals and ions, plus several isotopologues, were detected, the majority of them being recently revealed in comets C/1996 B2 (Hyakutake) and C/1995 O1 (Hale-Bopp). Among them, 6 molecules were detected for the first time (Bockelée-Morvan et al. 2000) in the course of a spectral survey conducted at radio wavelengths in comet Hale-Bopp with the CSO, the IRAM 30-m telescope and Plateau de Bure interferometer. In addition, many species were searched for unsuccessfully, some of them with stringent upper limits. We present here a review of these observations and further analysis of their results. This include: (i) confirmed detection of acetaldehyde $\left(\mathrm{CH}_{3} \mathrm{CHO}\right)$; (ii) limits on small molecules such as ketene $\left(\mathrm{H}_{2} \mathrm{CCO}\right)$ or methanimine $\left(\mathrm{CH}_{2} \mathrm{NH}\right)$; (iii) limits on the abundance ratios in homologous series such as $\mathrm{HC}_{5} \mathrm{~N} / \mathrm{HC}_{3} \mathrm{~N}$, ethanol/methanol, acetic acid/formic acid; (iv) searches for precursors of key cometary species such as atomic Na and $\mathrm{HNC}$; (v) constraints on more exotic species ranging from water dimer $\left(\mathrm{H}_{2} \mathrm{O}\right)_{2}$ to glycine; (vi) detection of the $\mathrm{H}_{2}^{34} \mathrm{~S}$ isotopic species and independent observations of HDO and DCN; (vii) limits on several other deuterated species; (viii) limits on several radicals and ions and a tentative detection of the $\mathrm{C}_{2} \mathrm{H}$ radical; (ix) the presence of unidentified lines. Typical abundance upper limits of $2-5 \times 10^{-4}$ relative to water are achieved for many species. Better upper limits are obtained for some linear molecules with high dipole moments. But more complex molecules such as dimethyl ether or glycine are poorly constrained. These results should give important clues to the chemical composition of cometary ices, to the formation mechanisms of cometary material, and to the chemical processes which occur in the inner coma.
\end{abstract}

Key words. astrochemistry - comets: general - comets: individuals: C/1995 O1 (Hale-Bopp) - line: identification radio lines: solar system - solar system: formation

\section{Introduction}

From radio spectroscopic observations of comets, more than 22 molecules, radicals and ions, plus several isotopologues, were detected, the majority of them being recently revealed in comets C/1996 B2 (Hyakutake) and C/1995 O1 (Hale-Bopp). This brought us new insights into the composition and nature of comets (Crovisier 1998; Despois 1999; Irvine et al. 2000a; Bockelée-Morvan et al. 2000, 2004).

We present here some of the results of a spectral survey at radio wavelengths made in comet $\mathrm{C} / 1995 \mathrm{O} 1$ (Hale-Bopp). We mainly concentrate on observations made around perihelion, from March to May 1997. In this period, the comet was at 0.91-1.4 AU from the Sun, at 1.3-2.2 AU from the Earth, and had a water outgassing rate from 5 to

Send offprint requests to: J. Crovisier,

e-mail: jacques.crovisier@obspm.fr
$11 \times 10^{30}$ mol. $\mathrm{s}^{-1}$. Three radio telescopes were used: the Caltech Submillimeter Observatory (CSO) on Mauna Kea (Hawaii), the Institut de radioastronomie millimétrique (IRAM) 30-m telescope on Pico Veleta (Spain) and the IRAM interferometer on Plateau de Bure (France; hereafter $\mathrm{PdBI}$ ), used here in the single-dish mode.

This survey resulted in the detection of most of the cometary molecules already known at radio wavelengths $(\mathrm{CO}$, $\mathrm{HCN}, \mathrm{CH}_{3} \mathrm{OH}, \mathrm{H}_{2} \mathrm{CO}, \mathrm{H}_{2} \mathrm{~S}, \mathrm{CS}, \mathrm{CH}_{3} \mathrm{CN}, \mathrm{HNC}$, OCS, HNCO) and of several new cometary molecules $\left(\mathrm{SO}, \mathrm{SO}_{2}, \mathrm{HC}_{3} \mathrm{~N}\right.$, $\left.\mathrm{NH}_{2} \mathrm{CHO}, \mathrm{HCOOH}, \mathrm{HCOOCH}{ }_{3}\right)$ and a new ion $\left(\mathrm{H}_{3} \mathrm{O}^{+}\right)$. This was reported by Biver et al. (1999), Lis et al. (1999) and Bockelée-Morvan et al. (1999, 2000). The present paper reports a further analysis of this survey focussed on the study of the non-detected molecules. During this analysis, two additional cometary molecules have been detected: acetaldehyde, which is discussed in Sect. 3.5, and ethylene glycol, which 
Table 1. Characteristics of the radio telescopes used for the spectral survey.

\begin{tabular}{lcccc}
\hline \hline Telescope & $\begin{array}{c}\text { Diam. } \\
{[\mathrm{m}]}\end{array}$ & $\begin{array}{c}\text { Freq. } \\
{[\mathrm{GHz}]}\end{array}$ & $\begin{array}{c}H P B W \\
{\left[{ }^{\prime \prime}\right]}\end{array}$ & $\begin{array}{c}\text { Beam } \\
\text { efficiency }\end{array}$ \\
\hline IRAM 30-m & 30 & 90 & 27 & 0.75 \\
& & 150 & 16 & 0.52 \\
& & 230 & 11 & 0.39 \\
IRAM PdBI & $5 \times 15$ & 115 & 42 & 0.83 \\
\multirow{2}{*}{ CSO } & & 230 & 21 & 0.58 \\
& \multirow{2}{*}{10} & 230 & 31 & 0.72 \\
& & 345 & 21 & 0.65 \\
\hline
\end{tabular}

is presented in a separate paper (Crovisier et al. 2004). We also report on the detection of the $\mathrm{H}_{2}^{34} \mathrm{~S}$ isotopic species and on detections of HDO and DCN (which were also detected at the James Clerk Maxwell Telescope, as previously reported by Meier et al. 1998a,b).

Upper limits from previous extensive searches at radio wavelengths were published by Crovisier et al. (1993) for comets C/1989 X1 (Austin) and C/1990 K1 (Levy), and by Lis et al. (1997) for comet C/1996 B2 (Hyakutake). Upper limits for sodium compounds searched for at the CSO in comet Hale-Bopp were also reported by Lis et al. (1999).

Section 2 describes the survey, its strategy of observation and the methods of analysis. The following sections will comment on the results molecule per molecule, for putative parent molecules (Sect. 3), isotopic species (Sect. 4), radicals and ions (Sect. 5). Section 6 is a general discussion of the results.

\section{Observations}

\subsection{The radio telescopes and spectrometers}

A description of the telescopes used in the present work is given in Table 1. The receivers and backends are described in more detail in Bockelée-Morvan et al. (2000). Observations were optimized by making the best use of parallel modes and of the versatility of the backends (several receivers with the IRAM 30-m, double sideband mode with the IRAM PdBI and CSO, several backends in parallel with all telescopes). Two kinds of spectra were obtained, most of the time simultaneously: a) highresolution spectra allowing to resolve the lines, but covering a small spectral range (e.g., 128 channels of $100 \mathrm{kHz}$ ); these spectra were aimed at specific lines; b) low-resolution spectra, covering a large spectral range (e.g., 500 channels of $1 \mathrm{MHz}$ ); these spectra were devoted to serendipitous searches.

\subsection{Strategy of observations}

A real spectral survey, i.e. the full coverage of a comprehensive spectral region, was not possible because of the large amount of observing time required to make sensitive observations, and of the limited period when the comet was bright and observable. We thus restricted our observations to selected spectral domains. These domains were chosen so that they encompass as many "interesting" lines as possible.

To help us in choosing the spectral domains to observe, we developed a computer programme (PAPSYNTHE) to make synthetic cometary spectra. This programme uses as input the JPL molecular database files (Pickett et al. 1998) and the following parameters: geocentric distance $\Delta$; heliocentric distance $r_{\mathrm{h}}$; telescope diameter; coma expansion velocity $V_{\exp }$; molecular rotational temperature $T_{\text {rot }}$; the list of cometary molecules with their hypothetical abundances relative to water and their photodissociation rates; the water production rate $Q\left[\mathrm{H}_{2} \mathrm{O}\right]$. The molecules are assumed to be at local thermodynamical equilibrium with a constant $T_{\text {rot }}$ throughout the coma and to have a parent molecule Haser distribution.

The predictions of PAPSYNTHE were used to select the most intense lines of a given species, and to spot combinations of lines of different species that could be observed with the same receiver setting. In many cases, it was possible to include in the spectral domain to be observed a line from an abundant species (such as $\mathrm{CH}_{3} \mathrm{OH}$ or $\mathrm{H}_{2} \mathrm{CO}$ ) which could be easily detected: this provided a direct validation of the observation (check of the telescope pointing and of the receiver tuning).

We estimate that, altogether, a spectral domain of $\approx 30 \mathrm{GHz}$ was covered during our observations, which corresponds to about $10 \%$ of the region between 85 and $375 \mathrm{GHz}$. It was found afterwards that strong transitions of several species, which were not considered in our primary choice, were serendipitously observed during our survey. We anticipate that several other species (radicals and ions), for which spectral data are still lacking, were also covered in these observations. Further work on our data is still awaiting molecular databases for further species.

\subsection{Principle of analysis}

As in our previous studies (Crovisier et al. 1993; Lis et al. 1997) we made an extensive use of the PAPSYNTHE software and of JPL and Cologne molecular databases (Pickett et al. 1998; Müller et al. 2001). For some species not included in these databases, or for which updated data are available $\left(\mathrm{CH}_{3} \mathrm{OCH}_{3}\right.$, $\mathrm{CH}_{3} \mathrm{CHO}, \mathrm{CH}_{3} \mathrm{OD} \ldots$ ), we converted the available molecular data files to the JPL format. For other species without available convenient electronic files (e.g., $\left.\left(\mathrm{H}_{2} \mathrm{O}\right)_{2}\right)$, a case-by-case study was necessary (and the analysis is still in progress).

Production rates are evaluated assuming a parent molecule Haser distribution. The expansion velocities $\left(V_{\exp }\right)$ and rotational temperatures $\left(T_{\text {rot }}\right)$ are interpolated from Table 2. The photodissociation rates, when available, are taken from Crovisier (1994) or Huebner et al. (1992) and are listed in Table 3. They are assumed to be $10^{-4} \mathrm{~s}^{-1}$ at $1 \mathrm{AU}$ when unknown. The relative production rates (relative to water - the so-called "abundances") are estimated using water production rates interpolated from Table 2. The line intensities or their upper limits are determined from the observed spectra by integrating over $[-2,+2] \mathrm{km} \mathrm{s}^{-1}$, which is expected to cover the full width for unblended lines. 
Table 2. Parameters of comet Hale-Bopp used to derive molecular abundances.

\begin{tabular}{rrrccc}
\hline \hline $\begin{array}{r}\text { Date } \\
\mathrm{dd} / \mathrm{mm} / \mathrm{yy}\end{array}$ & $\begin{array}{r}r_{\mathrm{h}} \\
{[\mathrm{AU}]}\end{array}$ & $\begin{array}{r}\Delta \\
{[\mathrm{AU}]}\end{array}$ & $\begin{array}{r}T_{\text {rot }} \\
{[\mathrm{K}]}\end{array}$ & $\begin{array}{c}V_{\exp } \\
{\left[\mathrm{km} \mathrm{s}^{-1}\right]}\end{array}$ & $\begin{array}{c}Q\left[\mathrm{H}_{2} \mathrm{O}\right] \\
{\left[10^{30} \mathrm{~s}^{-1}\right]}\end{array}$ \\
\hline $1 / 08 / 96$ & 3.58 & 2.73 & 25 & 0.60 & 0.2 \\
$15 / 02 / 97$ & 1.21 & 1.73 & 83 & 0.93 & 5.5 \\
$1 / 03 / 97$ & 1.07 & 1.49 & 96 & 0.98 & 8.0 \\
$15 / 03 / 97$ & 0.96 & 1.34 & 108 & 1.02 & 9.0 \\
$1 / 04 / 97$ & 0.91 & 1.35 & 114 & 1.05 & 10.0 \\
$15 / 04 / 97$ & 0.95 & 1.51 & 109 & 1.03 & 11.0 \\
$1 / 05 / 97$ & 1.06 & 1.77 & 97 & 0.98 & 8.0 \\
$15 / 05 / 97$ & 1.19 & 2.01 & 85 & 0.94 & 5.5 \\
$1 / 06 / 97$ & 1.39 & 2.27 & 72 & 0.88 & 4.0 \\
\hline
\end{tabular}

$T_{\text {rot }}$ and $V_{\text {exp }}$ are taken from Biver et al. (1999). $Q\left[\mathrm{H}_{2} \mathrm{O}\right]$ is from SWAN/SOHO Lyman $\alpha$ data (Combi et al. 2000) and from the observations of the $18-\mathrm{cm}$ line of $\mathrm{OH}$ at Nançay (Colom et al. 1999).

\section{Results: Various molecules}

For each molecule, a selection of the most significant observations are listed line by line in Tables 4-6. The summary of the resulting limits on the relative production rates is listed in Table 7.

\subsection{Water dimer $\left(\left(\mathrm{H}_{2} \mathrm{O}\right)_{2}\right)$}

An independent search for microwave lines of the water dimer $\left(\left(\mathrm{H}_{2} \mathrm{O}\right)_{2}\right)$ was made by Scherer et al. (1998) in comet Hale-Bopp. This search was restricted to centimetric lines observable at the Effelsberg radio telescope, which are not the most favourable transitions.

Analyses of the water dimer rotational spectrum were published by Coudert \& Hougen (1990) and Zwart et al. (1991). A comprehensive list of transitions was made available to us by Coudert (pers. comm.), upon which our search was based. The partition function and line strengths were evaluated as in Scherer et al. (1998). Our upper limit is $0.5 \%$ relative to water. This is an order of magnitude lower than the limit $(<6 \%)$ obtained by Scherer et al. from the observation of the $2_{0} E^{-}-1_{0} E^{+}$line at $24.284 \mathrm{GHz}$.

From theoretical predictions (Crifo \& Slanina 1991), water dimers are expected to have small abundances $\left(<10^{-5}\right.$ for comet Halley) and our limit is probably not significant.

\subsection{Hydrogen peroxide $\left(\mathrm{H}_{2} \mathrm{O}_{2}\right)$}

This molecule is not detected in the interstellar medium. Our upper limit is $0.03 \%$.

\subsection{Propyne $\left(\mathrm{CH}_{3} \mathrm{CCH}\right)$}

Propyne (aka methyl acetylene) is the smallest asymmetric hydrocarbon, with a dipole moment of $0.78 \mathrm{D}$. It can therefore be searched for by its rotational lines. It was sometimes proposed as a parent for the $\mathrm{C}_{3}$ radical (Krasnopolsky 1991; see also the
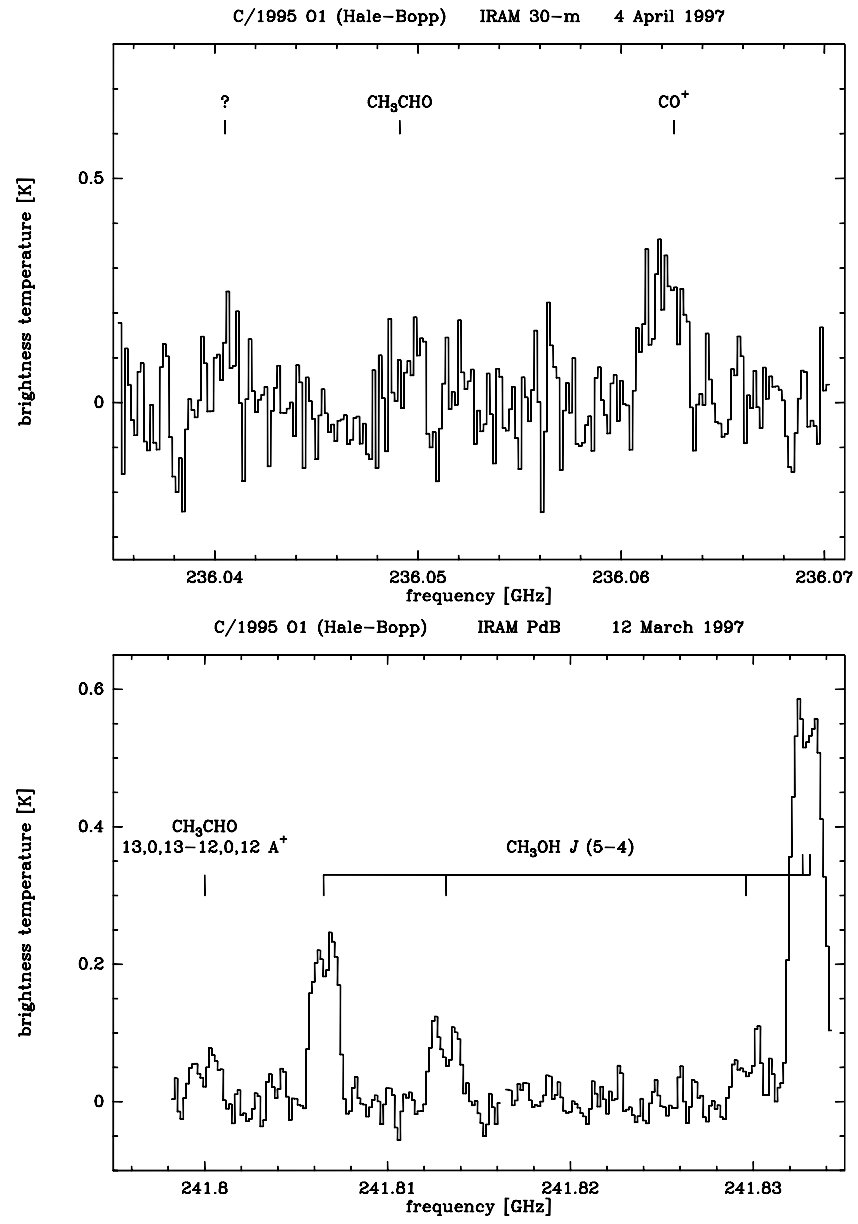

Fig. 1. Observations of acetaldehyde in comet C/1995 O1 Hale-Bopp. Top: a marginal line of acetaldehyde observed at IRAM $30-\mathrm{m}$ at $236.0491 \mathrm{GHz}$, together with a $\mathrm{CO}^{+}$line and an unidentified feature. Bottom: rotational lines of methanol and acetaldehyde observed at IRAM PdBI. The $x$-axis scale is the frequency in the principal sideband where the methanol lines appear. The acetaldehyde line is at $244.832 \mathrm{GHz}$ in the image sideband.

discussion by Helbert et al. 2000). Its abundance upper limit, however, $(0.045 \%)$ is slightly smaller than the $\mathrm{C}_{3}$ production rate $(\approx 0.06 \%$ from Schleicher et al. 1997).

\subsection{Ketene $\left(\mathrm{CH}_{2} \mathrm{CO}\right)$}

Our abundance upper limit is $0.045 \%$ for this molecule.

\subsection{Acetaldehyde $\left(\mathrm{CH}_{3} \mathrm{CHO}\right)$}

Molecular data are taken from Kleiner et al. (1996). At the beginning of the observations, we were not aware of the Kleiner et al. paper and our search for $\mathrm{CH}_{3} \mathrm{CHO}$ was based upon the JPL database, in which the frequencies were in error of several $\mathrm{MHz}$ for this species.

The detection of $\mathrm{CH}_{3} \mathrm{CHO}$ was confirmed during the present work, after the paper of Bockelée-Morvan et al. (2000) reporting the radio detection of other molecules was completed. A series of lines were marginally observed with the IRAM 30-m in April and May (one of them is shown in Fig. 1, 
Table 3. Photodissociation rates and dipole moments of the molecules investigated in the present work.

\begin{tabular}{|c|c|c|c|c|c|}
\hline \multirow[t]{2}{*}{ Molecule } & \multirow{2}{*}{$\begin{array}{c}\text { Photo rate } \\
{\left[\mathrm{s}^{-1}\right]}\end{array}$} & \multicolumn{3}{|c|}{ Dipole moment [D] } & \multirow[t]{2}{*}{ Reference } \\
\hline & & $\mu_{A}$ & $\mu_{B}$ & $\mu_{C}$ & \\
\hline$\left(\mathrm{H}_{2} \mathrm{O}\right)_{2}$ & - & 2.633 & $\approx 0$. & $\approx 0$. & $\mathrm{O}$ \\
\hline $\mathrm{H}_{2} \mathrm{O}_{2}$ & $1.3 \times 10^{-4}$ & 0.657 & 1.732 & - & JPL \\
\hline $\mathrm{CH}_{3} \mathrm{CCH}$ & $4.2 \times 10^{-5}$ & - & 0.780 & - & JPL \\
\hline $\mathrm{CH}_{2} \mathrm{CO}$ & $4.4 \times 10^{-4}$ & 1.422 & - & - & JPL \\
\hline $\mathrm{CH}_{3} \mathrm{CHO}$ & $7.5 \times 10^{-5}$ & 0.262 & 2.33 & - & $\mathrm{O}$ \\
\hline $\mathrm{c}-\mathrm{C}_{2} \mathrm{H}_{4} \mathrm{O}$ & - & - & 1.88 & - & CDMS \\
\hline $\mathrm{C}_{2} \mathrm{H}_{5} \mathrm{OH}$ trans & $1.8 \times 10^{-5}$ & 0.046 & 1.438 & - & JPL \\
\hline $\mathrm{CH}_{3} \mathrm{OCH}_{3}$ & $3.1 \times 10^{-5}$ & - & 1.302 & - & $\mathrm{O}$ \\
\hline $\mathrm{CH}_{3} \mathrm{COOH}$ & $5.1 \times 10^{-5}$ & 0.86 & 1.47 & - & $\mathrm{O}$ \\
\hline $\mathrm{CH}_{2} \mathrm{OHCHO}$ & - & 2.55 & 0.87 & - & CDMS \\
\hline $\mathrm{NH}_{2} \mathrm{CH}_{2} \mathrm{COOH} \mathrm{I}$ & - & 0.91 & 0.70 & - & JPL \\
\hline $\mathrm{HC}_{5} \mathrm{~N}$ & - & - & $(4.33)$ & - & JPL \\
\hline $\mathrm{C}_{2} \mathrm{H}_{5} \mathrm{CN}$ & - & 3.85 & 1.23 & - & JPL \\
\hline $\mathrm{CH}_{2} \mathrm{NH}$ & - & 1.325 & 1.530 & - & JPL \\
\hline $\mathrm{NH}_{2} \mathrm{CN}$ & - & 4.327 & - & 0.961 & JPL \\
\hline $\mathrm{NH}_{2} \mathrm{OH}$ & - & 0.598 & - & 0.60 & CDMS \\
\hline HCNO & - & - & 3.06 & - & $\mathrm{O}$ \\
\hline $\mathrm{N}_{2} \mathrm{O}$ & $5.9 \times 10^{-6}$ & - & 0.161 & - & JPL \\
\hline NS & - & - & 1.81 & - & JPL \\
\hline $\mathrm{H}_{2} \mathrm{CS}$ & - & 1.649 & - & - & JPL \\
\hline $\mathrm{CH}_{3} \mathrm{SH}$ & - & 1.312 & 0.758 & - & $\mathrm{O}$ \\
\hline $\mathrm{NaCl}$ & - & - & 8.97 & - & JPL \\
\hline $\mathrm{NaOH}$ & - & - & $(6.83)$ & - & JPL \\
\hline $\mathrm{PH}_{3}$ & $6.1 \times 10^{-5}$ & - & - & 0.574 & JPL \\
\hline HDO & $\left(1.3 \times 10^{-5}\right)$ & 0.657 & 1.732 & - & JPL \\
\hline DCN & $\left(1.5 \times 10^{-5}\right)$ & - & 2.984 & - & JPL \\
\hline HDCO & $\left(2.0 \times 10^{-4}\right)$ & 2.330 & 0.195 & - & JPL \\
\hline $\mathrm{CH}_{3} \mathrm{OD}$ & $\left(1.3 \times 10^{-5}\right)$ & 0.833 & 1.488 & - & $\mathrm{O}$ \\
\hline $\mathrm{CH}_{2} \mathrm{DOH}$ & $\left(1.3 \times 10^{-5}\right)$ & $(0.885)$ & (1.440) & - & $\mathrm{O}$ \\
\hline $\mathrm{NH}_{2} \mathrm{D}$ & $\left(1.3 \times 10^{-4}\right)$ & 0.18 & - & 1.463 & JPL \\
\hline HDS & $\left(2.5 \times 10^{-4}\right)$ & 0.470 & 0.974 & - & JPL \\
\hline $\mathrm{H}_{2}^{34} \mathrm{~S}$ & $\left(2.5 \times 10^{-4}\right)$ & - & (0.974) & - & $\mathrm{O}$ \\
\hline $\mathrm{SiC}$ & - & - & $(1.7)$ & - & JPL \\
\hline $\mathrm{SiO}$ & - & - & 3.098 & - & JPL \\
\hline $\mathrm{HCO}$ & - & 1.363 & 0.700 & - & JPL \\
\hline $\mathrm{C}_{2} \mathrm{H}$ & - & - & $(0.77)$ & - & JPL \\
\hline HNO & - & 1.03 & 1.31 & - & JPL \\
\hline $\mathrm{HCP}$ & - & - & 0.390 & - & JPL \\
\hline $\mathrm{HOCO}^{+}$ & - & $(2.0)$ & $(2.8)$ & - & JPL \\
\hline $\mathrm{H}_{2} \mathrm{COH}^{+}$ & - & (1.44) & (1.77) & - & CDMS \\
\hline $\mathrm{HCNH}^{+}$ & - & - & $(0.29)$ & - & CDMS \\
\hline $\mathrm{N}_{2} \mathrm{H}^{+}$ & - & - & 3.4 & - & JPL \\
\hline
\end{tabular}

Dipole moment values between parentheses are uncertain ( $\mathrm{ab}$ initio calculations). Photodestruction rates for isotopologues are assumed equal to those of the main isotopic species. The last column lists which molecular data set was used: "JPL" for the JPL data set, "CDMS" for the Cologne data set and "O" for others (see text).

top). The confirmation came from the serendipitous observation of the $13_{0,13}-12_{0,12} A^{+}$line together with methanol lines at PdBI (Fig. 1, bottom). This line is observed at the 7- $\sigma$ level, on the edge of the band, with a shape closely resembling those 
Table 4. Parameters of molecular lines searched in the present survey.

\begin{tabular}{|c|c|c|c|c|c|c|}
\hline \multirow[t]{2}{*}{ Transition } & \multirow{2}{*}{$\begin{array}{c}\text { Frequency } \\
{[\mathrm{GHz}]}\end{array}$} & \multirow[t]{2}{*}{ Telescope } & \multirow{2}{*}{$\begin{array}{r}\text { Date } \\
\mathrm{dd} / \mathrm{mm} / \mathrm{yy}\end{array}$} & \multirow{2}{*}{$\begin{array}{c}\text { Int. } \\
\text { [min] }\end{array}$} & \multicolumn{2}{|c|}{$\int T_{b} \mathrm{~d} v\left[\mathrm{mK} \mathrm{km} \mathrm{s}^{-1}\right]$} \\
\hline & & & & & Model & Observed \\
\hline \multicolumn{7}{|c|}{ Water dimer $\left(\mathrm{H}_{2} \mathrm{O}\right)_{2}$} \\
\hline $12_{1} E^{+}-11_{1} E^{-}$ & 146.9609 & IRAM 30-m & $8 / 04 / 97$ & 130 & 21 & $<105$ \\
\hline \multirow[t]{2}{*}{$12_{-2} E^{-}-11_{-2} E^{+}$} & 147.1302 & IRAM 30-m & $17 / 05 / 97$ & 102 & 9 & $<57$ \\
\hline & & IRAM 30-m & $22 / 05 / 97$ & 162 & 9 & $<51$ \\
\hline & & [CS (3-2) line sir & & & & \\
\hline \multirow[t]{2}{*}{$11_{0} A_{1}^{-}-10_{0} A_{1}^{+}$} & 157.1691 & IRAM 30-m & $5 / 04 / 97$ & 85 & 27 & $<165$ \\
\hline & & {$\left[\mathrm{CH}_{3} \mathrm{OH}\right.$ lines si } & & & & \\
\hline \multicolumn{7}{|c|}{ Hydrogen peroxide $\mathrm{H}_{2} \mathrm{O}_{2}$} \\
\hline $4_{1,3}-3_{0,3}$ & 143.7126 & IRAM 30-m & $21 / 05 / 97$ & $157 *$ & 252 & $<78$ \\
\hline $6_{1,5}-5_{0,5}$ & 251.9147 & $\mathrm{CSO}$ & $3 / 04 / 97$ & $18 *$ & 190 & $<90$ \\
\hline \multicolumn{7}{|c|}{ Propyne $\mathrm{CH}_{3} \mathrm{CCH}$} \\
\hline $13_{0}-12_{0}$ & 222.1670 & IRAM PdB & $18 / 03 / 97$ & 34 & 71 & $<23$ \\
\hline \multicolumn{7}{|c|}{ [and other lines of the $13-12$ series] } \\
\hline $14_{0}-13_{0}$ & 239.2522 & $\mathrm{CSO}$ & 27/03/97 & 32 & 37 & $<250$ \\
\hline \multicolumn{2}{|c|}{ [and other lines of the $14-13$ series] } & \multicolumn{5}{|c|}{ [simultaneous lines of $\mathrm{CH}_{3} \mathrm{CN}$ ] } \\
\hline \multicolumn{7}{|c|}{ Ketene $\mathrm{CH}_{2} \mathrm{CO}$} \\
\hline \multirow[t]{2}{*}{$11_{1,11}-10_{1,10}$} & 220.1782 & IRAM 30-m & $15 / 05 / 97$ & $50 *$ & 294 & $<323$ \\
\hline & & IRAM 30-m & $22 / 05 / 97$ & $135^{*}$ & & $<93$ \\
\hline \multirow[t]{2}{*}{$13_{1,13}-12_{1,12}$} & 260.1948 & $\mathrm{CSO}$ & $16 / 04 / 97$ & 10 & 51 & $<192$ \\
\hline & & $\mathrm{CSO}$ & $17 / 04 / 97$ & 44 & & $<220$ \\
\hline \multicolumn{7}{|c|}{ Acetaldehyde $\mathrm{CH}_{3} \mathrm{CHO}$} \\
\hline \multirow{2}{*}{$11_{1,10^{-}}-10_{1,9} A^{-}$} & 216.6302 & IRAM 30-m & $16 / 05 / 97$ & $45^{*}$ & 859 & $264 \pm 67$ \\
\hline & & IRAM 30-m & $22 / 05 / 97$ & $102 *$ & & $66 \pm 24$ \\
\hline $12_{2,11}-11_{2,10} A^{-}$ & 230.3019 & IRAM 30-m & $22 / 05 / 97$ & $27 *$ & 876 & $126 \pm 42$ \\
\hline & 226.5927 & IRAM 30-m & $11 / 04 / 97$ & $65^{*}$ & 944 & $175 \pm 66$ \\
\hline \multirow{2}{*}{$\begin{array}{l}12_{0,12}-11_{0,11} A \\
12_{1,11}-11_{1,10} A^{-}\end{array}$} & 236.0491 & IRAM 30-m & $4 / 04 / 97$ & $128 *$ & 1064 & $219 \pm 59$ \\
\hline & & $\mathrm{CSO}$ & $22 / 02 / 97$ & 22 & 268 & $<47$ \\
\hline $13_{2,1} 2-12_{2,11} A^{-}$ & 249.3239 & $\mathrm{CSO}$ & $22 / 04 / 97$ & 38 & 204 & $<50$ \\
\hline $13_{3,10}-12_{3,9} A^{-}$ & 251.4892 & $\mathrm{CSO}$ & $21 / 04 / 97$ & 32 & 182 & $<55$ \\
\hline $13_{0,13}-12_{0,12} A^{+}$ & 244.8322 & IRAM PdBI & $12 / 03 / 97$ & $16^{*}$ & 508 & $122 \pm 17$ \\
\hline & & [line near the bar & & & & \\
\hline \multirow[t]{2}{*}{$13_{0,13}-12_{0,12} E$} & 244.7892 & IRAM PdBI & $12 / 03 / 97$ & $16^{*}$ & 508 & - \\
\hline & & [line blended wit & 1 lines] & & & \\
\hline \multicolumn{7}{|c|}{ Ethylene oxide $\mathrm{c}-\mathrm{C}_{2} \mathrm{H}_{4} \mathrm{O}$} \\
\hline $8_{1,7}-7_{2,6}$ & 254.2317 & $\mathrm{CSO}$ & $21 / 04 / 97$ & 16 & 11 & $<95$ \\
\hline $8_{2,7}-7_{1,6}$ & 254.2357 & & & & 6 & $<95$ \\
\hline $9_{0,9}-8_{1,8}$ & 263.2925 & $\mathrm{CSO}$ & $8 / 04 / 97$ & 22 & 9 & $<50$ \\
\hline $9_{1,9}-8_{0,8}$ & 263.2925 & & & & 15 & \\
\hline \multicolumn{7}{|c|}{ Ethanol $\mathrm{C}_{2} \mathrm{H}_{5} \mathrm{OH}$ trans } \\
\hline $14_{1,14}-13_{0,13}$ & 235.9833 & IRAM 30-m & 4/04/97 & $113^{*}$ & 290 & $<170$ \\
\hline $4_{4,1}-3_{3,0}$ & 252.9514 & $\mathrm{CSO}$ & $23 / 04 / 97$ & $40 *$ & 43 & $<75$ \\
\hline $4_{4,0}-3_{3,1}$ & 252.9524 & & & & 43 & \\
\hline & & [Simultaneous C & & & & \\
\hline
\end{tabular}

Each entry gives:

- the transition denomination;

- the frequency (in GHz);

- the telescope;

- the date of the observation;

- the integration time (“*” denotes observations made at high spectral resolution);

- the expected line intensity (in $\mathrm{mK} \mathrm{km} \mathrm{s}^{-1}$ ) from the PAPSYNTHE model for a production rate of $10^{28} \mathrm{~s}^{-1}$;

- the 3- $\sigma$ upper limit (in $\mathrm{mK} \mathrm{km} \mathrm{s}^{-1}$ ) or the line area with its $1-\sigma$ error in case of (marginal) detection. 
Table 4. continued.

\begin{tabular}{|c|c|c|c|c|c|c|}
\hline \multirow[t]{2}{*}{ Transition } & \multirow{2}{*}{$\begin{array}{c}\text { Frequency } \\
{[\mathrm{GHz}]}\end{array}$} & \multirow[t]{2}{*}{ Telescope } & \multirow{2}{*}{$\begin{array}{r}\text { Date } \\
\mathrm{dd} / \mathrm{mm} / \mathrm{yy}\end{array}$} & \multirow{2}{*}{$\begin{array}{c}\text { Int. } \\
{[\mathrm{min}]}\end{array}$} & \multicolumn{2}{|c|}{$\int T_{b} \mathrm{~d} v\left[\mathrm{mK} \mathrm{km} \mathrm{s}^{-1}\right]$} \\
\hline & & & & & Model & Observed \\
\hline \multicolumn{7}{|c|}{ Dimethyl ether $\mathrm{CH}_{3} \mathrm{OCH}_{3}$} \\
\hline $7_{1,7}-6_{0,6}$ & 147.0249 & IRAM 30-m & $8 / 04 / 97$ & $130 *$ & 41 & $216 \pm 54$ \\
\hline $10_{4,6}-10_{3,7}$ & 203.5703 & IRAM 30-m & $3 / 04 / 97$ & $150^{*}$ & 59 & $<153$ \\
\hline $4_{3,2}-3_{2,2}$ & 222.4226 & $\mathrm{CSO}$ & $23 / 04 / 97$ & $24 *$ & 10 & $<100$ \\
\hline $4_{3,1}-3_{2,2}$ & 222.4337 & $\mathrm{CSO}$ & $24 / 04 / 97$ & 6 & 10 & $<180$ \\
\hline \multirow[t]{4}{*}{$12_{1,12}-11_{0,11}$} & 225.5991 & $\mathrm{CSO}$ & 23/04/97 & $24^{*}$ & 34 & $243 \pm 69$ \\
\hline & & IRAM PdB & $16 / 03 / 97$ & 28 & & $<80$ \\
\hline & & IRAM 30-m & $17 / 05 / 97$ & 244 & 97 & $<100$ \\
\hline & & \multicolumn{3}{|c|}{ [Simultaneous $\mathrm{H}_{2} \mathrm{CO}$ line at $225.697 \mathrm{GHz}$ ] } & & \\
\hline \multicolumn{7}{|c|}{ Acetic acid $\mathrm{CH}_{3} \mathrm{COOH}$} \\
\hline $18_{*, 16}-17_{*, 15} A$ & 216.8116 & IRAM 30-m & $21 / 05 / 97$ & $102 *$ & 120 & $<150$ \\
\hline $19_{*, 17}-18_{*, 16} A$ & 227.4542 & IRAM 30-m & $5 / 04 / 97$ & $165^{*}$ & 150 & $<89$ \\
\hline \multicolumn{7}{|c|}{ Glycolaldehyde $\mathrm{CH}_{2} \mathrm{OHCHO}$} \\
\hline $14_{0,14}-13_{1,13}$ & 143.6409 & IRAM 30-m & $21 / 05 / 97$ & 120 & 57 & $<75$ \\
\hline $14_{1,14}-13_{0,13}$ & 143.7656 & & & & 57 & $<75$ \\
\hline $13_{2,12}-12_{1,11}$ & 146.0193 & IRAM 30-m & $17 / 05 / 97$ & 105 & 40 & $<55$ \\
\hline $20_{0,20}-19_{1,19}$ & 203.2668 & IRAM 30-m & 03/04/97 & 152 & 229 & $320 \pm 120$ \\
\hline $20_{1,20}-19_{0,19}$ & 203.2701 & & & & 229 & \\
\hline $20_{3,17}-19_{4,16}$ & 218.2605 & IRAM 30-m & $21 / 05 / 97$ & 55 & 44 & $<150$ \\
\hline $7_{6,2}-6_{5,1}$ & 220.1966 & IRAM 30-m & $22 / 05 / 97$ & 135 & 87 & $<75$ \\
\hline $7_{6,1}-6_{5,2}$ & 220.1969 & & & & 87 & \\
\hline \multicolumn{7}{|c|}{ Glycine I $\mathrm{NH}_{2} \mathrm{CH}_{2} \mathrm{COOH}$} \\
\hline \multirow[t]{2}{*}{$35_{*, 31}-34_{*, 32}$} & 222.611 & $\mathrm{CSO}$ & 23/04/97 & 24 & 16 & $<135$ \\
\hline & & $\mathrm{CSO}$ & $24 / 04 / 97$ & 6 & & $<180$ \\
\hline $12_{11, *}-11_{10, *}$ & 227.3609 & IRAM 30-m & 5/04/97 & 310 & 35 & $<66$ \\
\hline $33_{7, *}-32_{7, *}$ & 227.3628 & & & & 35 & $<66$ \\
\hline $\begin{array}{c}33_{14, *}-32_{14, *} \\
\text { [and many others] }\end{array}$ & \multicolumn{5}{|c|}{ [and many others] } & $<66$ \\
\hline \multicolumn{7}{|c|}{ Cyanodiacetylene $\mathrm{HC}_{5} \mathrm{~N}$} \\
\hline \multirow[t]{2}{*}{$34-33$} & 90.5262 & IRAM PdB & $13 / 03 / 97$ & 24 & 360 & $<30$ \\
\hline & & IRAM 30-m & $5 / 04 / 97$ & $295^{*}$ & 1125 & $<35$ \\
\hline $40-39$ & 106.4994 & IRAM 30-m & $4 / 04 / 97$ & $150 *$ & 1600 & $<53$ \\
\hline $54-53$ & 143.7663 & IRAM 30-m & $21 / 05 / 97$ & $157^{*}$ & 1295 & $<64$ \\
\hline 59-58 & 157.0743 & IRAM 30-m & $5 / 04 / 97$ & 85 & 2240 & $<180$ \\
\hline \multicolumn{7}{|c|}{ Ethyl cyanide $\mathrm{C}_{2} \mathrm{H}_{5} \mathrm{CN}$} \\
\hline $25_{, 3,22}-24_{, 3,21}$ & 227.7808 & IRAM 30-m & $5 / 04 / 97$ & 310 & 704 & $<66$ \\
\hline \multicolumn{7}{|c|}{ Methanimine $\mathrm{CH}_{2} \mathrm{NH}$} \\
\hline $1_{1,1}-0_{0,0}$ & 225.5548 & IRAM 30-m & $17 / 05 / 97$ & 244 & 290 & $<65$ \\
\hline $6_{1,5}-6_{0,6}$ & 226.5484 & IRAM 30-m & $11 / 04 / 97$ & $65^{*}$ & 545 & $<190$ \\
\hline $6_{1,6}-5_{1,5}$ & 251.4212 & $\mathrm{CSO}$ & $21 / 04 / 97$ & 32 & 115 & $<55$ \\
\hline $4_{1,3}-3_{1,2}$ & 266.2701 & $\mathrm{CSO}$ & 8/04/97 & $44 *$ & 220 & $<75$ \\
\hline
\end{tabular}

of the methanol lines in the same spectrum. The corresponding $13_{0,13}-12_{0,12} E$ line at $244.789 \mathrm{GHz}$, which was observed on the same day, is unfortunately blended with strong methanol lines.

$\mathrm{CH}_{3} \mathrm{CHO}$ is next to $\mathrm{H}_{2} \mathrm{CO}$ in the aldehyde homologous series. We measure $Q\left[\mathrm{CH}_{3} \mathrm{CHO}\right]=0.025 \%$ whereas $Q\left[\mathrm{H}_{2} \mathrm{CO}\right]$ was $1.1 \%$ (Bockelée-Morvan et al. 2000). Thus $Q\left[\mathrm{CH}_{3} \mathrm{CHO}\right] / Q\left[\mathrm{H}_{2} \mathrm{CO}\right]$ is only $\approx 1 / 50$. However, this should be mitigated by the fact that most of cometary formaldehyde is coming from a distributed source, the fraction really directly coming from nucleus ices being less than $6 \%$ of the total $\mathrm{H}_{2} \mathrm{CO}$ (Wink et al. 1999). $\left[\mathrm{CH}_{3} \mathrm{CHO}\right] /\left[\mathrm{H}_{2} \mathrm{CO}\right]$ could then be higher than $1 / 3$ in nucleus ices.

From mass spectroscopy investigations of $1 \mathrm{P} /$ Halley, Altwegg et al. (1999) reported the "probable" detection of acetaldehyde (or its isomers) with an abundance of $0.5 \%$, much higher than our value for comet Hale-Bopp. 
Table 4. continued.

\begin{tabular}{|c|c|c|c|c|c|c|}
\hline \multirow[t]{2}{*}{ Transition } & \multirow{2}{*}{$\begin{array}{c}\text { Frequency } \\
{[\mathrm{GHz}]}\end{array}$} & \multirow[t]{2}{*}{ Telescope } & \multirow{2}{*}{$\begin{array}{r}\text { Date } \\
\mathrm{dd} / \mathrm{mm} / \mathrm{yy}\end{array}$} & \multirow{2}{*}{$\begin{array}{c}\text { Int. } \\
{[\mathrm{min}]}\end{array}$} & \multicolumn{2}{|c|}{$\int T_{b} \mathrm{~d} v\left[\mathrm{mK} \mathrm{km} \mathrm{s}^{-1}\right]$} \\
\hline & & & & & Model & Observed \\
\hline \multicolumn{7}{|c|}{ Cyanamide $\mathrm{NH}_{2} \mathrm{CN}$} \\
\hline $11_{1,11}-10_{1,10}$ & 218.4618 & IRAM 30-m & $3 / 04 / 97$ & $53^{*}$ & 4760 & $<200$ \\
\hline \multicolumn{7}{|c|}{ Hydroxylamine $\mathrm{NH}_{2} \mathrm{OH}$} \\
\hline $5_{1,5}-4_{1,4}$ & 251.6774 & $\mathrm{CSO}$ & $5 / 04 / 97$ & 68 & 28 & $<70$ \\
\hline $5_{0,5}-4_{0,4}$ & 251.8385 & & & & 31 & $<70$ \\
\hline \multicolumn{7}{|c|}{ Fulminic acid $\mathrm{HCNO}$} \\
\hline $11-10$ & 252.2965 & $\mathrm{CSO}$ & $22 / 04 / 97$ & $38 *$ & 3800 & $<60$ \\
\hline \multicolumn{7}{|c|}{ Nitrous oxide $\mathrm{N}_{2} \mathrm{O}$} \\
\hline $10-9$ & 251.2116 & $\mathrm{CSO}$ & $21 / 04 / 97$ & $32 *$ & 29 & $<67$ \\
\hline $11-10$ & 276.3278 & $\mathrm{CSO}$ & $7 / 04 / 97$ & 32 & 35 & $<92$ \\
\hline \multicolumn{7}{|l|}{ Radical NS } \\
\hline $5 / 2-3 / 2 c$ & 115.153 & $\begin{array}{l}\text { IRAM 30-m } \\
\text { [simultaneous CC }\end{array}$ & $12 / 05 / 97$ & 15 & 174 & $<420$ \\
\hline $11 / 2-9 / 2$ & 253.968 & $\begin{array}{l}\mathrm{CSO} \\
\text { [simultaneous } \mathrm{Cr}\end{array}$ & $21 / 04 / 97$ & 32 & 785 & $<82$ \\
\hline $15 / 2-13 / 2 e$ & 345.823 & JCMT & $22 / 03 / 97$ & & 4380 & $=640 \pm 110$ \\
\hline \multirow[t]{2}{*}{$15 / 2-13 / 2 f$} & 346.220 & JCMT & $22 / 03 / 97$ & & 4380 & $=480 \pm 60$ \\
\hline & & [For comparison: & by Irvine et al. 2 & & & \\
\hline \multicolumn{7}{|c|}{ Thioformaldehyde $\mathrm{H}_{2} \mathrm{CS}$} \\
\hline \multirow[t]{3}{*}{$8_{1,8}-7_{1,7}$} & 270.5207 & $\mathrm{CSO}$ & $19 / 03 / 97$ & $16^{*}$ & 440 & $<200$ \\
\hline & & & $27 / 03 / 97$ & 22 & & $<150$ \\
\hline & & [simultaneous $\mathrm{HC}$ & very close] & & & \\
\hline $9_{1,9}-8_{1,8}$ & 304.3060 & $\begin{array}{l}\mathrm{CSO} \\
\text { [simultaneous } \mathrm{CI}\end{array}$ & 4/04/97 & 40 & 600 & $<240$ \\
\hline \multirow[t]{2}{*}{$7_{1,6}-6_{1,5}$} & 244.0478 & NRAO 12-m & $23 / 03 / 97$ & & 435 & $=167 \pm 39$ \\
\hline & & [For comparison: & by Woodney et & & & \\
\hline \multicolumn{7}{|c|}{ Methyl mercaptan $\mathrm{CH}_{3} \mathrm{SH}$} \\
\hline $9_{0}-8_{0} A^{+}$ & 227.3264 & IRAM 30-m & $5 / 04 / 97$ & 310 & 190 & $<24$ \\
\hline $9_{2}-8_{2} A^{-}$ & 227.5314 & IRAM 30-m & $5 / 04 / 97$ & $310^{*}$ & 155 & $<72$ \\
\hline other lines & & IRAM PdB & $6 / 03 / 97$ & 8 & & $<80$ \\
\hline \multirow[t]{2}{*}{ of the 9-8 series } & & IRAM PdB & $11 / 03 / 97$ & 27 & & $<60$ \\
\hline & & [simultaneous $\mathrm{HC}$ & $227.4189 \mathrm{GHz}$ & & & \\
\hline \multirow[t]{2}{*}{$10_{4}-9_{4} A^{-}$} & 252.8415 & $\mathrm{CSO}$ & 23/04/97 & 40 & & $<45$ \\
\hline & & [simultaneous $\mathrm{Cr}$ & & & & \\
\hline \multicolumn{7}{|c|}{ Phosphine $\mathrm{PH}_{3}$} \\
\hline $1_{0}-0_{0}$ & 266.9447 & $\mathrm{CSO}$ & $2 / 08 / 96$ & $96^{*}$ & 13 & $<21$ \\
\hline
\end{tabular}

\subsection{Ethylene oxide $\left(c-\mathrm{C}_{2} \mathrm{H}_{4} \mathrm{O}\right)$}

Ethylene oxide (aka oxirane) is an isomer of acetaldehyde. The upper limit we obtain is $0.2 \%$. Thus, $\left[\left(\mathrm{c}-\mathrm{C}_{2} \mathrm{H}_{4} \mathrm{O}\right] /\left[\mathrm{CH}_{3} \mathrm{CHO}\right]<\right.$ 10 , which is not a stringent limit compared to the ratio $\left[\left(\mathrm{c}-\mathrm{C}_{2} \mathrm{H}_{4} \mathrm{O}\right] /\left[\mathrm{CH}_{3} \mathrm{CHO}\right]=0.1-0.4\right.$ observed in interstellar hot cores (Nummelin et al. 1998).

\subsection{Ethanol $\left(\mathrm{C}_{2} \mathrm{H}_{5} \mathrm{OH}\right)$}

The ethanol ground state is the trans conformer. Two other conformers, the gauche + and gauche -, are present at about $57 \mathrm{~K}$ above the ground state and are indeed observed in the interstellar medium (Pearson et al. 1998). Our limit $(<0.05 \%)$ refers to the trans conformer only. At $100 \mathrm{~K}$, the total $\mathrm{C}_{2} \mathrm{H}_{5} \mathrm{OH}$ abundance is about 2.1 times that of the ground state conformer. Thus, the limit for total ethanol is $0.1 \%$. 
Table 5. Parameters of molecular lines searched in the present survey: deuterated and isotopic species.

\begin{tabular}{|c|c|c|c|c|c|c|}
\hline \multirow[t]{2}{*}{ Transition } & \multirow{2}{*}{$\begin{array}{c}\text { Frequency } \\
{[\mathrm{GHz}]}\end{array}$} & \multirow[t]{2}{*}{ Telescope } & \multirow{2}{*}{$\begin{array}{r}\text { Date } \\
\mathrm{dd} / \mathrm{mm} / \mathrm{yy}\end{array}$} & \multirow{2}{*}{$\begin{array}{c}\text { Int. } \\
{[\mathrm{min}]}\end{array}$} & \multicolumn{2}{|c|}{$\int T_{b} \mathrm{~d} v\left[\mathrm{mK} \mathrm{km} \mathrm{s}^{-1}\right]$} \\
\hline & & & & & Model & Observed \\
\hline \multicolumn{7}{|c|}{ Deuterated water $\mathrm{HDO}$} \\
\hline \multirow{2}{*}{$3_{1,2}-2_{2,1}$} & 225.8967 & IRAM PdB & $9,16 / 03 / 97$ & 40 & - & $105 \pm 21$ \\
\hline & & IRAM 30-m & $17 / 05 / 97$ & $244 *$ & - & $90 \pm 20$ \\
\hline $2_{1,1}-2_{1,2}$ & 241.5616 & $\mathrm{CSO}$ & $27 / 03 / 97$ & $85^{*}$ & - & $<55$ \\
\hline \multicolumn{7}{|c|}{ Deuterated hydrogen cyanide DCN } \\
\hline $2-1$ & 144.8280 & IRAM 30-m & $29 / 05 / 97$ & $145^{*}$ & - & $<105$ \\
\hline \multirow[t]{2}{*}{$3-2$} & 217.2385 & IRAM 30-m & $9,10 / 04 / 97$ & $253^{*}$ & - & $217 \pm 57$ \\
\hline & & [simultaneous $\mathrm{Cl}$ & & & & \\
\hline \multirow[t]{2}{*}{$5-4$} & 362.0465 & $\mathrm{CSO}$ & 6/04/97 & $43^{*}$ & - & $<335$ \\
\hline & & & $24 / 04 / 97$ & $13^{*}$ & - & $<680$ \\
\hline \multicolumn{7}{|c|}{ Deuterated formaldehyde $\mathrm{HDCO}$} \\
\hline $1_{1,1}-0_{0,0}$ & 227.6681 & IRAM 30-m & $5 / 04 / 97$ & 310 & 4 & $<90$ \\
\hline $7_{1,6}-7_{0,7}$ & 252.7264 & $\mathrm{CSO}$ & $23 / 04 / 97$ & 40 & 2 & $<70$ \\
\hline \multirow[t]{2}{*}{$6_{0,6}-5_{1,5}$} & 252.9639 & & & & 2 & $<70$ \\
\hline & & [Simultaneous C & & & & \\
\hline \multirow[t]{2}{*}{$4_{2,2}-3_{2,1}$} & 259.0349 & $\mathrm{CSO}$ & $21 / 04 / 97$ & 10 & 298 & $<150$ \\
\hline & & [Simultaneous C & SO lines] & & & \\
\hline \multicolumn{7}{|c|}{ Deuterated methanol $\mathrm{CH}_{3} \mathrm{OD}$} \\
\hline $5_{1} A^{-}-5_{0} A^{+}$ & 143.7417 & IRAM 30-m & $21 / 05 / 97$ & $120^{*}$ & 178 & $<62$ \\
\hline $5_{0} A^{+}-4_{0} A^{+}$ & 226.5387 & IRAM 30-m & $11 / 04 / 97$ & 65 & 160 & $<210$ \\
\hline 5-4 E series & 226.277 & & & & 266 & $280 \pm 71$ \\
\hline $5_{-2} E-5_{-1} E$ & 270.5814 & $\mathrm{CSO}$ & $27 / 03 / 97$ & 22 & 86 & $<270$ \\
\hline $6_{2} A^{+}-6_{1} A^{-}$ & 307.3682 & $\mathrm{CSO}$ & 4/04/97 & 40 & 122 & $<250$ \\
\hline \multicolumn{7}{|c|}{ Deuterated methanol $\mathrm{CH}_{2} \mathrm{DOH}$} \\
\hline $5_{1,5}-4_{1,4} e_{0}$ & 220.0719 & IRAM 30-m & $22 / 05 / 97$ & 135 & 82 & $<75$ \\
\hline $5_{1,4}-4_{1,3} e_{1}$ & 225.6676 & IRAM 30-m & $17 / 05 / 97$ & 244 & 62 & $<60$ \\
\hline $5_{1,4}-4_{1,3} e_{0}$ & 226.8184 & IRAM 30-m & $11 / 04 / 97$ & 65 & 153 & $<89$ \\
\hline \multicolumn{7}{|c|}{ Deuterated ammonia $\mathrm{NH}_{2} \mathrm{D}$} \\
\hline \multirow[t]{2}{*}{$3_{2,2}-3_{1,2}$} & 216.5626 & IRAM 30-m & $16 / 05 / 97$ & 45 & 180 & $<90$ \\
\hline & & & $29 / 05 / 97$ & 155 & 186 & $<60$ \\
\hline \multicolumn{7}{|c|}{ Deuterated hydrogen sulphide HDS } \\
\hline $1_{0,1}-0_{0,0}$ & 244.5556 & $\mathrm{CSO}$ & $27 / 03 / 97$ & 86 & 12 & $<35$ \\
\hline \multicolumn{7}{|c|}{ Hydrogen sulfide $\mathrm{H}_{2} \mathrm{~S},{ }^{34} \mathrm{~S}$ variety } \\
\hline $1_{1,0}-1_{0,1}$ & 167.9105 & IRAM 30-m & $9-10 / 11 / 96$ & $160 *$ & - & $107 \pm 24$ \\
\hline
\end{tabular}

See description in the footnotes of Table 4.

Comparing with the methanol production rate $(2.4 \%$ as reported by Bockelée-Morvan et al. 2000), we note that $Q\left[\mathrm{C}_{2} \mathrm{H}_{5} \mathrm{OH}\right] / Q\left[\mathrm{CH}_{3} \mathrm{OH}\right]<1 / 25$.

\subsection{Dimethyl ether $\left(\mathrm{CH}_{3} \mathrm{OCH}_{3}\right)$}

Molecular data are taken from Groner et al. (1998). The observed lines are blends of $A A, E E, A E$ and $E A$ transitions. The table lists the frequencies for the stronger $E E$ component and the intensities for the sum of the components. Our results are puzzling. There are two hints of detections, each at the 4- $\sigma$ level, which, if real, would correspond to an abundance of $0.5-0.7 \%$. However, the 17 May observation at IRAM 30-m (together with a strong formaldehyde line) puts a stringent upper limit of $0.1 \%$. We have adopted in Table 7 a conservative upper limit of $0.5 \%$.

\subsection{Acetic acid $\left(\mathrm{CH}_{3} \mathrm{COOH}\right)$}

The search for acetic acid was based upon molecular data from Wlodarczak \& Demaison (1988 and pers. comm.). A more comprehensive database is now available (Ilyushin et al. 2001, 2003; I. Kleiner, pers. comm.) which was used in our analysis.

The upper limit we obtain is $0.06 \%$. This is not much lower than the abundance of formic acid $(0.09 \%$; Bockelée-Morvan et al. 2000). 
Table 6. Parameters of molecular lines searched in the present survey: radicals and ions.

\begin{tabular}{|c|c|c|c|c|c|c|}
\hline \multirow[t]{2}{*}{ Transition } & \multirow{2}{*}{$\begin{array}{c}\text { Frequency } \\
{[\mathrm{GHz}]}\end{array}$} & \multirow[t]{2}{*}{ Telescope } & \multirow{2}{*}{$\begin{array}{r}\text { Date } \\
\mathrm{dd} / \mathrm{mm} / \mathrm{yy}\end{array}$} & \multirow{2}{*}{$\begin{array}{c}\text { Int. } \\
\text { [min] }\end{array}$} & \multicolumn{2}{|c|}{$\int T_{b} \mathrm{~d} v\left[\mathrm{mK} \mathrm{km} \mathrm{s}^{-1}\right]$} \\
\hline & & & & & Model & Observed \\
\hline \multicolumn{7}{|l|}{ Radical SiC } \\
\hline${ }^{3} \Pi_{2} J(6-5) e, f$ & 236.2876 & $\mathrm{CSO}$ & $27 / 03 / 97$ & 32 & 615 & $<250$ \\
\hline \multicolumn{7}{|l|}{ Radical $\mathrm{SiO}$} \\
\hline $5-4$ & 217.1050 & IRAM 30-m & $9,10 / 04 / 97$ & 253 & 18000 & $<250$ \\
\hline $7-6$ & 303.9270 & $\mathrm{CSO}$ & $4 / 04 / 97$ & 40 & 9000 & $<250$ \\
\hline \multicolumn{7}{|l|}{ Radical HCO } \\
\hline \multirow[t]{2}{*}{$3_{0,3}-2_{0,2}$} & 260.0603 & $\mathrm{CSO}$ & $16 / 04 / 97$ & 10 & 120 & $<190$ \\
\hline & & $\mathrm{CSO}$ & $17 / 04 / 97$ & 44 & & $<220$ \\
\hline \multicolumn{7}{|l|}{ Ethynyl radical $\mathrm{C}_{2} \mathrm{H}$} \\
\hline$N(3-2) J(7 / 2-5 / 2) F(4-3)$ & 262.0042 & $\mathrm{CSO}$ & $21 / 04 / 97$ & 10 & \multicolumn{2}{|c|}{ see text } \\
\hline$N(3-2) J(7 / 2-5 / 2) F(2-2)$ & 262.0065 & & & & & \\
\hline$N(3-2) J(5 / 2-3 / 2) F(3-2)$ & 262.0650 & & & & & \\
\hline$N(3-2) J(5 / 2-3 / 2) F(2-1)$ & 262.0675 & & & & & \\
\hline \multicolumn{7}{|l|}{ Nitroxyl HNO } \\
\hline $3_{0,3}-2_{0,2}$ & 244.3641 & $\mathrm{CSO}$ & $27 / 03 / 97$ & $85^{*}$ & & $<52$ \\
\hline \multicolumn{7}{|l|}{ Methylidynephosphine $\mathrm{HCP}$} \\
\hline $7-6$ & 279.6347 & $\mathrm{CSO}$ & $7 / 04 / 97$ & 32 & 115 & $<100$ \\
\hline \multicolumn{7}{|c|}{ Protonated carbon dioxide $\mathrm{HOCO}^{+}$} \\
\hline \multirow[t]{2}{*}{$5_{0,5}-4_{0,4}$} & 106.9136 & IRAM PdB & $20 / 03 / 97$ & $34 *$ & 85 & $<21$ \\
\hline & & IRAM PdB & $21 / 03 / 97$ & $58 *$ & & $<19$ \\
\hline \multicolumn{7}{|c|}{ Protonated formaldehyde $\mathrm{H}_{2} \mathrm{COH}^{+}$} \\
\hline $1_{1,0}-1_{0,1}$ & 168.4011 & IRAM 30-m & $3 / 04 / 97$ & $224 *$ & & $<180$ \\
\hline \multicolumn{7}{|c|}{ Protonated hydrogen cyanide $\mathrm{HCNH}^{+}$} \\
\hline $3-2$ & 222.3293 & IRAM 30-m & $26 / 05 / 97$ & $53^{*}$ & 86 & $<500$ \\
\hline \multicolumn{7}{|l|}{ Protonated dinitrogen $\mathrm{N}_{2} \mathrm{H}^{+}$} \\
\hline $3-2$ & 279.5117 & $\mathrm{CSO}$ & $7 / 04 / 97$ & 32 & 9400 & $<100$ \\
\hline
\end{tabular}

See description in the footnotes of Table 4.

\subsection{Glycolaldehyde $\left(\mathrm{CH}_{2} \mathrm{OHCHO}\right)$}

A line of $\mathrm{CH}_{2} \mathrm{OHCHO}$ is marginally present at the $2.7-\sigma$ level, which would correspond to an abundance of $0.07 \%$. But the absence of other lines sets up an upper limit of $0.04 \%$.

See also the discussion in Crovisier et al. (2004), where $\mathrm{CH}_{2} \mathrm{OHCHO}$ is compared to ethylene glycol $\left(\mathrm{HOCH}_{2} \mathrm{CH}_{2} \mathrm{OH}\right)$, which is detected with an abundance of $0.25 \%$.

Methyl formate $\left(\mathrm{HCOOCH}_{3}\right)$, acetic acid and glycolaldehyde are isomers. Of these species, only the first one is detected in the comet, with an abundance of $0.08 \%$ (Bockelée-Morvan et al. 2000), the others having slightly lower abundance upper limits.

\subsection{Glycine $\left(\mathrm{NH}_{2} \mathrm{CH}_{2} \mathrm{COOH}\right)$}

Glycine $\left(\mathrm{NH}_{2} \mathrm{CH}_{2} \mathrm{COOH}\right)$ is the simplest amino acid. Considering its interest for astrobiology, this molecule has been actively searched for in the interstellar medium (Miao et al. 1994; Combes et al. 1996; Snyder 1997; Ceccarelli et al. 2000; Hollis et al. 2003 and references therein). Definite detection has been claimed by Kuan et al. (2003). Molecular data for conformers I and II of glycine are available from Lovas et al. (1995) and are listed in the JPL database. The present upper limit has been evaluated for conformer I. Conformer II has a stronger dipole moment, but its equilibrium energy is $700 \mathrm{~cm}^{-1}$ higher. From laboratory measurement, Ehrenfreund et al. (2001a) have evaluated the lifetime of glycine (and of other amino acids) to be of the order of a few hours in cometary conditions (at $1 \mathrm{AU}$ ), which is similar to our default value of $10000 \mathrm{~s}$.

Our best upper limits come from three lines expected with similar intensity around $227 \mathrm{GHz}$. The limit for individual lines is $0.19 \%$. Co-adding the three lines would provide a limit of $0.11 \%$. However, one of the lines has a frequency uncertainty of $\approx 3 \mathrm{MHz}$. Co-adding the two other lines provides an upper limit of $0.14 \%$, which is the value listed in Table 7 .

This upper limit ( $\approx 0.15 \%$ in number relative to water) corresponds to $\approx 0.3 \%$ in mass in cometary ice, or $0.05 \%$ in mass in cometary material if we assume a dust-to-ice ratio of 5 (estimates of the dust-to-gas ratio in this comet range from 2 to 10 ; Grün et al. 2001; Weiler et al. 2003). In CI and CM type carbonaceous chondrites, which could be akin to cometary material, glycine is among the most abundant amino acids, the other 
Table 7. Summary of molecular upper limits in comet Hale-Bopp derived from the present work.

\begin{tabular}{|c|c|}
\hline Molecule & {$[\mathrm{X}] /\left[\mathrm{H}_{2} \mathrm{O}\right]$} \\
\hline $\mathrm{H}_{2} \mathrm{O}$ & 100 \\
\hline$\left(\mathrm{H}_{2} \mathrm{O}\right)_{2}$ & $<0.5$ \\
\hline $\mathrm{H}_{2} \mathrm{O}_{2}$ & $<0.03$ \\
\hline $\mathrm{CH}_{3} \mathrm{CCH}$ & $<0.045$ \\
\hline $\mathrm{CH}_{2} \mathrm{CO}$ & $<0.032$ \\
\hline $\mathrm{CH}_{3} \mathrm{CHO}$ & $=0.025$ \\
\hline $\mathrm{c}-\mathrm{C}_{2} \mathrm{H}_{4} \mathrm{O}$ & $<0.20$ \\
\hline $\mathrm{C}_{2} \mathrm{H}_{5} \mathrm{OH}$ & $<0.10$ \\
\hline $\mathrm{CH}_{3} \mathrm{OCH}_{3}$ & $<0.45$ \\
\hline $\mathrm{CH}_{3} \mathrm{COOH}$ & $<0.06$ \\
\hline $\mathrm{CH}_{2} \mathrm{OHCHO}$ & $<0.04$ \\
\hline $\mathrm{NH}_{2} \mathrm{CH}_{2} \mathrm{COOH} \mathrm{I}$ & $<0.15$ \\
\hline $\mathrm{HC}_{5} \mathrm{~N}$ & $<0.003$ \\
\hline $\mathrm{C}_{2} \mathrm{H}_{5} \mathrm{CN}$ & $<0.010$ \\
\hline $\mathrm{CH}_{2} \mathrm{NH}$ & $<0.032$ \\
\hline $\mathrm{NH}_{2} \mathrm{CN}$ & $<0.004$ \\
\hline $\mathrm{NH}_{2} \mathrm{OH}$ & $<0.25$ \\
\hline HCNO & $<0.0016$ \\
\hline $\mathrm{N}_{2} \mathrm{O}$ & $<0.23$ \\
\hline NS & $<0.01$ \\
\hline $\mathrm{H}_{2} \mathrm{CS}$ & $<0.04$ \\
\hline $\mathrm{CH}_{3} \mathrm{SH}$ & $<0.05$ \\
\hline $\mathrm{NaOH}$ & $<0.0003$ \\
\hline $\mathrm{NaCl}$ & $<0.0008$ \\
\hline $\mathrm{PH}_{3}$ & $<0.16$ \\
\hline $\mathrm{HDCO}$ & $<0.05$ \\
\hline $\mathrm{CH}_{3} \mathrm{OD}$ & $<0.07$ \\
\hline $\mathrm{CH}_{2} \mathrm{DOH}$ & $<0.06$ \\
\hline $\mathrm{NH}_{2} \mathrm{D}$ & $<0.08$ \\
\hline HDS & $<0.3$ \\
\hline
\end{tabular}

All upper limits are given at the $3-\sigma$ level.

ones being alanine, $\gamma$-amino- $n$-butyric acid and aminoisobutyric acid. The glycine concentration is $\approx 7 \times 10^{-7}$ in mass in the CI-type meteorites Orgueil and Ivuna (Ehrenfreund et al. $2001 \mathrm{~b}), \approx 2.5 \times 10^{-6}$ in the CM-type meteorites Murchison and Murray (Cronin \& Chang 1993). In this respect, our limit in comet Hale-Bopp is hardly significant. On the other hand, the abundance of glycine measured by Kuan et al. (2003) in the Galactic Centre (Sgr B2) and in Orion KL is comparable to other minor gas phase species such as formic acid. We note that our limit on glycine, which is at the level of $\approx 1.5$ the abundance of $\mathrm{HCOOH}$, is quite relevant in this context.

\subsection{Cyanodiacetylene $\left(\mathrm{HC}_{5} \mathrm{~N}\right)$}

Our upper limit for $\mathrm{HC}_{5} \mathrm{~N}$ is $0.003 \%$, whereas the abundances of $\mathrm{HCN}$ and $\mathrm{HC}_{3} \mathrm{~N}$ are 0.25 and 0.020 , respectively (Bockelée-Morvan et al. 2000). Thus, $\left[\mathrm{HC}_{5} \mathrm{~N}\right] /\left[\mathrm{HC}_{3} \mathrm{~N}\right]<0.14$ whereas $\left[\mathrm{HC}_{3} \mathrm{~N}\right] /[\mathrm{HCN}]=0.08$, showing the continuous decrease of the abundance of cyanopolyynes with increasing carbon chain. See Sect. 6 and Fig. 6.

\subsection{Methanimine $\left(\mathrm{CH}_{2} \mathrm{NH}\right)$}

Our upper limit for $\mathrm{CH}_{2} \mathrm{NH}$ is $0.03 \%$.

This molecule was proposed as a possible parent for HNC (the unstable isomer of HCN) by Irvine et al. (1998), but their search for $\mathrm{CH}_{2} \mathrm{NH}$ was inconclusive. The origin of cometary $\mathrm{HNC}$ is a mystery. Its abundance relative to $\mathrm{HCN}$ varies from comet to comet and increases with decreasing heliocentric distance (Biver et al. 1999, 2002; Irvine et al. 1999). Chemical models fail to explain its production from the conversion of HCN in the coma (Rodgers \& Charnley 2001).

Our upper limit corresponds to $Q\left[\mathrm{CH}_{2} \mathrm{NH}\right]<3 . \times 10^{27} \mathrm{~s}^{-1}$ near perihelion. At that time, $Q[\mathrm{HNC}]$ was about $3-4 \times 10^{27} \mathrm{~s}^{-1}$ (Biver et al. 1999), assuming a parent molecule distribution; if this species is indeed coming from an extended source, its production rate is underestimated. Thus, even if the yield for the $\mathrm{CH}_{2} \mathrm{NH} \rightarrow \mathrm{HNC}+2 \mathrm{H}$ photodissociation channel is unity, our observation seems to exclude the production of all the cometary HNC from methanimine ${ }^{1}$.

A related $\mathrm{CHN}$ molecule is methylamine $\left(\mathrm{CH}_{3} \mathrm{NH}_{2}\right)$. A comprehensive data set of microwave lines is still lacking for this species. The published lines of this molecule were not covered in our survey.

\subsection{Ethyl cyanide $\left(\mathrm{C}_{2} \mathrm{H}_{5} \mathrm{CN}\right)$}

Our upper limit $(0.01 \%)$ is half the abundances of the detected carbon-chain cyanides $\mathrm{CH}_{3} \mathrm{CN}$ and $\mathrm{HC}_{3} \mathrm{~N}(0.02 \%$ from Bockelée-Morvan et al. 2000).

\subsection{Cyanamide $\left(\mathrm{NH}_{2} \mathrm{CN}\right)$}

Our upper limit is $0.004 \%$. This very low value is due to the high dipole moment of the molecule.

\subsection{Hydroxylamine $\left(\mathrm{NH}_{2} \mathrm{OH}\right)$}

Although not detected in the interstellar medium, hydroxylamine is suspected to be an important constituent of interstellar ices (Charnley et al. 2001). Our upper limit is $0.25 \%$, somewhat below the abundance of ammonia $(0.7 \%$ from Bird et al. 1999).

\subsection{Fulminic acid ( $\mathrm{HCNO})$}

Fulminic acid is an isomer of isocyanic acid (HNCO, observed with an abundance of $0.1 \%$; Bockelée-Morvan et al. 2000). Molecular data are from Winnewisser \& Winnewisser (1971). This species has not been detected in the interstellar medium.

\footnotetext{
1 Unless the lifetime of $\mathrm{CH}_{2} \mathrm{NH}$ is significantly smaller than our assumed value of $10^{4} \mathrm{~s}$, in which case we would have underestimated its production rate.
} 
Our upper limit corresponds to an isomeric ratio $Q[\mathrm{HCNO}] / Q[\mathrm{HNCO}]<1 / 60$. The good limit is due to the high dipole moment $(3.06 \mathrm{D})$ of this linear molecule.

\subsection{Nitrous oxide $\mathrm{N}_{2} \mathrm{O}$}

Nitrogen oxides have small dipole moments and their radio lines cannot be easily observed. Our limit for $\mathrm{N}_{2} \mathrm{O}(0.23 \%)$ is thus not stringent. Unfortunately, nitric oxide $\mathrm{NO}$ was not covered in the present survey.

\subsection{NS radical}

The NS radical was detected by Irvine et al. (2000b) in comet Hale-Bopp on 22 March at the James Clerk Maxwell Telescope (JCMT) $(J(15 / 2-13 / 2) e$ and $f$ transitions at $346 \mathrm{GHz})$. No plausible parent could be found which would produce this radical by photodissociation. Chemical models do not predict any significant production of NS in the coma (Canaves et al. 2002). The presence of this radical is thus a puzzle. A direct production from cometary ices has been invoked (Irvine et al. 2000b).

We could not confirm this detection (serendipitous observation of the $J(11 / 2-9 / 2)$ transition at $254 \mathrm{GHz}$ with the CSO on 21 April). Our upper limit (0.01\%) is about twice lower than the abundance derived by Irvine et al. (assuming in both cases a parent molecule distribution). One should consider, however, the unknown spatial distribution of this radical, the fact that different lines were observed, and possible non-thermal excitation.

\subsection{Thioformaldehyde $\left(\mathrm{H}_{2} \mathrm{CS}\right)$}

Thioformaldehyde $\left(\mathrm{H}_{2} \mathrm{CS}\right)$ was detected in comet Hale-Bopp by Woodney et al. (1999) with the National Radio Astronomical Observatory (NRAO) 12-m telescope at Kitt Peak. This detection relies on the observation of a single line with a limited $S / N$.

We could not confirm this detection. Our upper limit is $0.04 \%$ and is similar to the abundance $(0.038 \%)$ we derive from the Woodney et al. observation, using the same model parameters. Woodney (pers. comm. quoted by Bockelée-Morvan et al. 2000) derived an abundance of $0.02 \%$, but an abundance of $0.05 \%$ is reported in Woodney (2000). The photodissociation rate of $\mathrm{H}_{2} \mathrm{CS}$ is unknown. Woodney used 2. $\times 10^{-4} \mathrm{~s}^{-1}$, i.e. the same as that of $\mathrm{H}_{2} \mathrm{CO}$, whereas we used our default value of $10^{-4} \mathrm{~s}^{-1}$.

\subsection{Methyl mercaptan $\left(\mathrm{CH}_{3} \mathrm{SH}\right)$}

Molecular data are taken from Bettens et al. (1999). The upper limit is $0.05 \%$, which corresponds to $Q\left[\mathrm{CH}_{3} \mathrm{SH}\right] / Q\left[\mathrm{CH}_{3} \mathrm{OH}\right]<$ 0.02 , not significantly smaller than the cosmic abundance ratio $[\mathrm{S}] /[\mathrm{O}]=0.032 \pm 0.10$ (Grevesse \& Sauval 1998).

\subsection{Phosphine $\left(\mathrm{PH}_{3}\right)$}

Phosphine is detected in the atmospheres of Jupiter and Saturn, but not in interstellar clouds. No phosphorous species has ever been observed in comets.

Phosphine was searched for through its fundamental rotational line $1_{0}-0_{0}$ at $266.945 \mathrm{GHz}$ at the CSO. The observation took place on 2 August 1996 when the comet was still at 3.6 AU from the Sun and was not repeated, unfortunately. The upper limit is $Q\left[\mathrm{PH}_{3}\right]<3.2 \times 10^{26} \mathrm{~s}^{-1}$. This corresponds to $Q\left[\mathrm{PH}_{3}\right] / Q\left[\mathrm{H}_{2} \mathrm{O}\right]<0.0016$, which is not significant compared to the cosmic elemental abundance ratio $[\mathrm{P}] /[\mathrm{O}]=$ $4.2 \pm 0.8 \times 10^{-4}$ (Grevesse \& Sauval 1998).

However, $\mathrm{PH}_{3}$ is a very volatile species. It sublimates at an equilibrium temperature of $41 \mathrm{~K}$ under cometary conditions, comparable to that of the hypervolatiles $\mathrm{CO}(24 \mathrm{~K}), \mathrm{CH}_{4}(31 \mathrm{~K})$ or $\mathrm{C}_{2} \mathrm{H}_{6}(44 \mathrm{~K})$. At $r_{\mathrm{h}}=3.6 \mathrm{AU}$, the sublimation of water is inhibited by the low equilibrium temperature of the cometary nucleus, whereas that of the hypervolatiles is not. If we rescale the $\left[\mathrm{PH}_{3}\right] /\left[\mathrm{H}_{2} \mathrm{O}\right]$ ratio according to the $[\mathrm{CO}] /\left[\mathrm{H}_{2} \mathrm{O}\right]$ evolution with heliocentric distance (as measured by Biver et al. 1999), we would obtain $Q\left[\mathrm{PH}_{3}\right] / Q\left[\mathrm{H}_{2} \mathrm{O}\right]<1.6 \times 10^{-4}$ at $r_{\mathrm{h}} \approx 1 \mathrm{AU}$, now smaller than the cosmic $[\mathrm{P}] /[\mathrm{H}]$ ratio.

\subsection{Sodium species ( $\mathrm{NaCl}$ and $\mathrm{NaOH})$}

Atomic sodium in comets is conspicuous in the coma (or, for comet Hale-Bopp as a "third type" tail) through its $\mathrm{D}_{1}$ and $\mathrm{D}_{2}$ lines observed in comets at $r_{\mathrm{h}}<1.5 \mathrm{AU}$. Its origin, however, is still a mystery. Direct desorption from dust grains or degradation of parent molecules have been invoked. Since the $[\mathrm{Na}] /[\mathrm{O}]$ cosmic ratio is $1 / 300$ (Grevesse \& Sauval 1998) and the Na production rate observed in the comet was only $5 \times 10^{-6}$ relative to water (Cremonese et al. 1997), most of the cometary sodium must remain trapped in refractories.

The upper limits listed in Table 7 for $\mathrm{NaOH}$ and $\mathrm{NaCl}$ are those reported in Lis et al. (1999) and the detail of the observations is not repeated here. The limits are very low because of the very large dipole moments of these linear molecules (6.8 and 9.0 D, respectively). They are comparable to the Na production rate observed in the comet, so that no definite conclusion can be drawn from our observations on the nature of the $\mathrm{Na}$ source in comets. Furthermore, these limits were evaluated assuming lifetimes of $10^{4} \mathrm{~s}$ for these molecules. The real lifetimes are likely to be much shorter (Cremonese et al. 2002), in which case our upper limits are underestimated.

\section{Results: Isotopic species}

The enrichment of deuterium in cometary and interstellar molecules is characteristic of the fractionation which occurred during the formation of these molecules ${ }^{2}$. Up to now, HDO and DCN are the only deuterated molecules detected in comets (Bockelée-Morvan et al. 1998; Meier et al. 1998a,b). For comprehensive discussions of this topic, see Meier \& Owen (1999)

\footnotetext{
${ }^{2}$ Note that the $[\mathrm{D}] /[\mathrm{H}]$ ratio in a molecule $\mathrm{H}_{n} \mathrm{R}$ is $\frac{1}{n} \frac{\left[\mathrm{H}_{n-1} \mathrm{DR}\right]}{\left[\mathrm{H}_{n} \mathrm{R}\right]}$, not $\frac{\left[\mathrm{H}_{n-1} \mathrm{DR}\right]}{\left[\mathrm{H}_{n} \mathrm{R}\right]}$. Thus, $[\mathrm{D}] /[\mathrm{H}]_{\mathrm{H}_{2} \mathrm{O}}=0.5[\mathrm{HDO}] /\left[\mathrm{H}_{2} \mathrm{O}\right]$.
} 
Table 8. $[\mathrm{D}] /[\mathrm{H}]$ ratios in deuterated molecules in interstellar clouds and in comets.

\begin{tabular}{lcccc}
\hline \hline Molecule & $\begin{array}{r}\text { TMC-1 } \\
(10 \mathrm{~K})\end{array}$ & $\begin{array}{c}\text { OMC-1 } \\
(\sim 60 \mathrm{~K})\end{array}$ & $\begin{array}{c}\text { Hot cores } \\
(\sim 200 \mathrm{~K})\end{array}$ & Comets \\
\hline $\mathrm{HDO}$ & & & $>0.002$ & 0.0003 \\
$\mathrm{DCN}$ & 0.023 & 0.02 & 0.005 & 0.002 \\
$\mathrm{NH}_{2} \mathrm{D}$ & $<0.02$ & & 0.003 & $<0.04$ \\
$\mathrm{HDCO}$ & 0.015 & 0.02 & 0.14 & $<0.05$ \\
$\mathrm{CH}_{3} \mathrm{OD}$ & & & 0.03 & $<0.03$ \\
$\mathrm{CH}_{2} \mathrm{DOH}$ & & & 0.04 & $<0.008$ \\
$\mathrm{HDS}$ & & & $<0.001$ & $<0.2$ \\
\hline
\end{tabular}

Values for TMC-1, OMC-1 and hot cores are from Millar (2000) (from Hatchell et al. 1999 for HDS). Values for comets are from Bockelée-Morvan et al. (1998) and Meier et al. (1998a) for HDO, from Meier et al. (1998b) for DCN, and from the present work for upper limits.

and Altwegg \& Bockelée-Morvan (2003). [D]/[H] in cometary water was found to be $3 \times 10^{-4}$ in three comets (1P/Halley, C/1996 B2 (Hyakutake), C/1995 O1 (Hale-Bopp)), enriched by a factor of $\approx 10$ over the protosolar value. $[\mathrm{D}] /[\mathrm{H}]$ in $\mathrm{HCN}$ was still higher $\left(2 \times 10^{-3}\right)$ in comet Hale-Bopp. The deuterium enrichment could be even higher in other molecules, as is observed in interstellar clouds (Table 8).

\subsection{Deuterated water (HDO)}

HDO was detected in comet Hale-Bopp at the JCMT through its $1_{10}-0_{00}$ submillimetric line at $465 \mathrm{GHz}$, as was reported by Meier et al. (1998a). The observation of this line from the ground requires exceptionally good weather conditions.

Millimetric lines of HDO between low-energy levels are the $1_{10}-1_{11}$ line at $80.578 \mathrm{GHz}$ (which was then unfortunately not accessible to the IRAM receivers), the $3_{12}-2_{21}$ line at $225.897 \mathrm{GHz}$, and the $2_{11}-2_{12}$ line at $241.562 \mathrm{GHz}$. These lines are much weaker (by a factor of 10 or more) than the submillimetric lines of HDO. The $242 \mathrm{GHz}$ line was observed together with $5_{K}-4_{K}$ methanol lines at the CSO on 27 March, but was not detected. The $226 \mathrm{GHz}$ line was serendipitously detected at the edge of a low-resolution backend of the PdB interferometer on 9 and 16 March (Fig. 2, top). Despite the low velocity resolution and the signal-to-noise ratio of the line of $\approx 5$ only, confidence in the detection comes from the presence of the line in the two independent observations. This line is also present at the 4.5- $\sigma$ level in a high-resolution spectrum obtained on 17 May at the IRAM 30-m (Fig. 2, bottom).

The HDO production rates were computed using an excitation model which includes solar IR pumping of the vibrational bands and collisional excitation by electrons and neutrals (see Bockelée-Morvan et al. 1998; Meier et al. 1998a). Inferred $Q[\mathrm{HDO}]$ values are $9.4 \pm 1.9 \times 10^{27},<3.5 \times 10^{27}$, and $4.7 \pm 1.1 \times 10^{27} \mathrm{~s}^{-1}$ for 9 and 16 March, 27 March, and
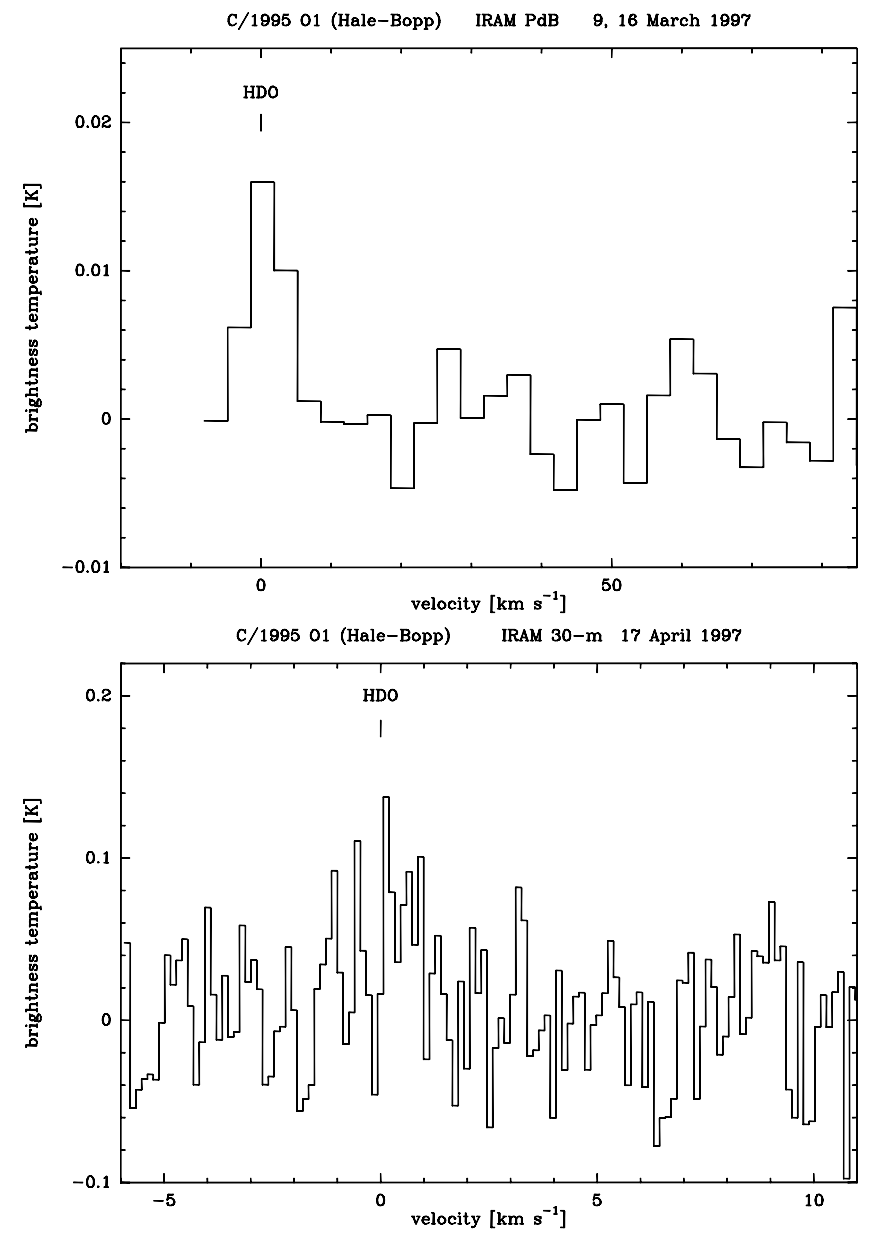

Fig. 2. The $3_{12}-2_{21}$ line of $\mathrm{HDO}$ at $225.897 \mathrm{GHz}$ observed at the PdBI in comet Hale-Bopp (top) and at the IRAM 30-m telescope (bottom).

17 May, respectively. This translates into $[\mathrm{D}] /[\mathrm{H}]_{\mathrm{H}_{2} \mathrm{O}}=5.2 \pm$ $1.2 \times 10^{-4},<1.8 \times 10^{-4}$, and $=4.1 \pm 1.0 \times 10^{-4}$, respectively. The uncertainties given in these numbers include a $10 \%$ uncertainty in $Q\left(\left[\mathrm{H}_{2} \mathrm{O}\right]\right)$ values. For comparison, Meier et al. (1998a) deduced $[\mathrm{D}] /[\mathrm{H}]_{\mathrm{H}_{2} \mathrm{O}}=3.3 \pm 0.8 \times 10^{-4}$ from the $465 \mathrm{GHz}$ line observed on 5 April, using the same modelling for HDO excitation. Therefore, the measurements obtained from the $226 \mathrm{GHz}$ and $465 \mathrm{GHz}$ lines agree within the error bars.

On the other hand, the low upper limit of $1.8 \times 10^{-4}$ we derive from the $242 \mathrm{GHz}$ line is puzzling. The simultaneous observation of $\mathrm{CH}_{3} \mathrm{OH}$ lines provides a methanol production rate consistent with contemporary independent measurements, thereby excluding a decrease of cometary activity at the very moment of the HDO observations. Model calculations show that the derived $Q[\mathrm{HDO}]$ is not very sensitive to assumed model parameters. So, we do not have any evident explanation for this conflicting result.

\subsection{Deuterated hydrogen cyanide (DCN)}

We searched for DCN through its $J(2-1)$ and (3-2) transitions at IRAM 30-m, and its $J(5-4)$ transition at the CSO. A tentative detection at the 4- $\sigma$ level was obtained near perihelion for the $J(3-2)$ line. It is reported in Table 5 and shown in 


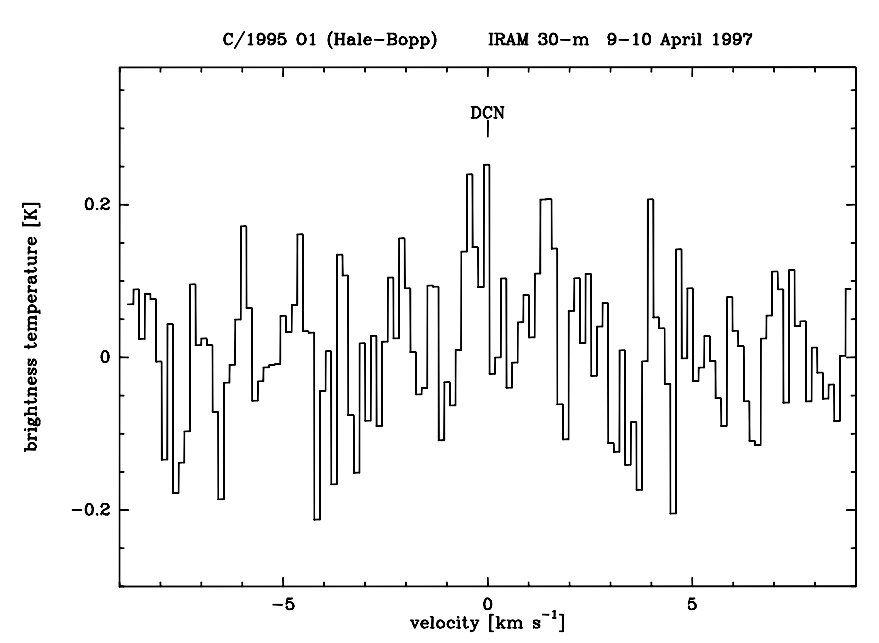

Fig. 3. The $J(3-2)$ line of DCN at $217.238 \mathrm{GHz}$ observed at the IRAM 30-m telescope in comet Hale-Bopp.

Fig. 3. It corresponds to $Q[\mathrm{DCN}]=7.9 \pm 2.1 \times 10^{25} \mathrm{~s}^{-1}$ and, compared to the simultaneous measurement of $Q[\mathrm{HCN}]$, to $[\mathrm{D}] /[\mathrm{H}]_{\mathrm{HCN}}=2.3 \pm 0.6 \times 10^{-3}$. For consistency, we have used the same modelling (described by Biver et al. 1999) for HCN and DCN, instead of PAPSYNTHE. This value is in excellent agreement with the measurement of Meier et al. (1998b) (2.3 \pm $\left.0.4 \times 10^{-3}\right)$. The upper limits for undetected lines are also higher than this value $\left([\mathrm{D}] /[\mathrm{H}]<7.7 \times 10^{-3}\right.$ and $<3.6 \times 10^{-3}$ for the $J(2-1)$ and $J(5-4)$ lines, respectively).

\subsection{Deuterated formaldehyde (HDCO)}

HDCO has two dipole moment components, $\mu_{A}=2.32 \mathrm{D}$ and $\mu_{B}=0.18 \mathrm{D}$, corresponding to $a$-type and $b$-type transitions. Hence, $a$-type transitions are two orders of magnitude stronger than $b$-type transitions. Unfortunately, most of the transitions which were searched for were $b$-type. An $a$-type transition $\left(4_{22}-3_{21}\right)$ was, however, observed serendipitously at the CSO, but the integration time was short and the limit is not very good. Our upper limit on HDCO comes from this observation. $\mathrm{H}_{2} \mathrm{CO}$ (and so should be HDCO) is observed to come, in part, from a distributed source of unknown nature (Colom et al. 1992; Bockelée-Morvan et al. 2000). This hampers the determination of the production rates of $\mathrm{H}_{2} \mathrm{CO}$ and HDCO. Our determination $[\mathrm{HDCO}] /\left[\mathrm{H}_{2} \mathrm{CO}\right]<0.1$, corresponding to $[\mathrm{D}] /[\mathrm{H}]<0.05$, however, is based upon the comparison of the column densities and does not suffer from this difficulty.

\subsection{Deuterated methanol $\left(\mathrm{CH}_{3} \mathrm{OD}, \mathrm{CH}_{2} \mathrm{DOH}\right)$}

The molecular data for $\mathrm{CH}_{3} \mathrm{OD}$ are taken from Anderson et al. (1988). A computer file of the $\mathrm{CH}_{3} \mathrm{OD}$ transitions, provided by Herbst (pers. comm.) was converted to the JPL catalogue format. Our upper limit is based upon the $5_{1}-5_{0} A$ line at 143.741 GHz observed at the IRAM 30-m.

For $\mathrm{CH}_{2} \mathrm{DOH}$ we also used a list of transitions provided by Herbst (pers. comm.), described in Parise et al. (2002). Note that if the deuterium atom can replace with the same probability the four hydrogen atoms of $\mathrm{CH}_{3} \mathrm{OH}$ (as expected from models involving grain chemistry;
Charnley et al. 1997), one would expect $\left[\mathrm{CH}_{2} \mathrm{DOH}\right]=3$ $\left[\mathrm{CH}_{3} \mathrm{OD}\right]$. The observation of $\mathrm{CH}_{2} \mathrm{DOH}$ is thus more sensitive for the measurement of the $[\mathrm{D}] /[\mathrm{H}]$ ratio. Jacq et al. (1993) observed $\left[\mathrm{CH}_{2} \mathrm{DOH}\right] /\left[\mathrm{CH}_{3} \mathrm{OD}\right]=1.1-1.5$ in Orion-IRc2, whereas Parise et al. (2002) observed $\left[\mathrm{CH}_{2} \mathrm{DOH}\right] /\left[\mathrm{CH}_{3} \mathrm{OD}\right]=$ $20 \pm 14$ in the protostar IRAS 162931-2422. We obtain $[\mathrm{D}] /[\mathrm{H}]_{\mathrm{CH}_{3} \mathrm{OH}}<0.03$ from $\mathrm{CH}_{3} \mathrm{OD},<0.008$ from $\mathrm{CH}_{2} \mathrm{DOH}$. For comparison, Eberhardt et al. (1994) measured $[\mathrm{D}] /[\mathrm{H}]_{\mathrm{CH}_{3} \mathrm{OH}}<0.01$ from mass spectroscopy in comet Halley (mass spectroscopy cannot distinguish $\mathrm{CH}_{2} \mathrm{DOH}$ from $\left.\mathrm{CH}_{3} \mathrm{OD}\right)$.

\subsection{Other deuterated species $\left(\mathrm{NH}_{2} \mathrm{D}, \mathrm{HDS}\right)$}

$\mathrm{NH}_{2} \mathrm{D}$ has not been searched for on purpose. A line serendipitously observed at IRAM 30-m does not provide a very stringent limit $\left([\mathrm{D}] /[\mathrm{H}]_{\mathrm{NH}_{3}}<0.04\right.$; however, $[\mathrm{D}] /[\mathrm{H}]_{\mathrm{NH}_{3}}$ as high as 0.05 has been recently observed in $\mathrm{NH}_{3}$ in dark clouds by Saito et al. 2000).

For HDS, only the fundamental transition $1_{01}-0_{00}$ at $244.556 \mathrm{GHz}$ was searched for, at the CSO. The upper limit is not very significant $\left([\mathrm{D}] /[\mathrm{H}]_{\mathrm{H}_{2} \mathrm{~S}}<0.2\right)$.

Although no detection could be achieved, the upper limits obtained on $\mathrm{HDCO}, \mathrm{CH}_{3} \mathrm{OD}$ and $\mathrm{CH}_{2} \mathrm{DOH}$, which are the most significant, show that these molecules are less deuterium enriched in comets than in interstellar clouds, confirming the result obtained for HDO and DCN (Table 8). This possibility results from reprocessing in the Solar Nebula, as proposed by Hersant et al. (2001): gas-phase $\mathrm{H}_{2} \mathrm{O}$ and $\mathrm{HCN}$, originating from ices infalling from the presolar cloud, underwent isotopic exchanges with $\mathrm{H}_{2}$ in the inner Solar Nebula; reprocessed gas then diffused by turbulence to the outer regions where comets formed. The rate of isotopic exchange is species dependent and less efficient for $\mathrm{HCN}$.

\subsection{Other isotopic species $\left(\mathrm{H}_{2}{ }^{34} \mathrm{~S}\right)$}

Jewitt et al. (1997) measured $\left[{ }^{12} \mathrm{C}\right] /\left[{ }^{13} \mathrm{C}\right],\left[{ }^{14} \mathrm{~N}\right] /\left[{ }^{15} \mathrm{~N}\right]$ and $\left[{ }^{32} \mathrm{~S}\right] /\left[{ }^{34} \mathrm{~S}\right]$ ratios from the observations of radio lines of $\mathrm{H}^{13} \mathrm{CN}, \mathrm{HC}^{15} \mathrm{~N}$ and $\mathrm{C}^{34} \mathrm{~S}$ with the JCMT. Our observation of $\mathrm{H}^{13} \mathrm{CN}$ at the CSO is discussed by Lis et al. (1999).

$\mathrm{H}_{2}^{34} \mathrm{~S}$ was searched for and tentatively detected at the 4- $\sigma$ level on 9 and 10 Nov. 1996 at IRAM through its $1_{10}-1_{01}$ line at $167.910 \mathrm{GHz}$ (Fig. 4). The molecular data are taken from Helminger et al. (1973). A direct comparison with the corresponding line of $\mathrm{H}_{2}^{32} \mathrm{~S}$ (which is optically thin) observed at the same moment gives $\left[{ }^{32} \mathrm{~S}\right] /\left[{ }^{34} \mathrm{~S}\right]=16.5 \pm 3.5$. This is lower than the value $\left[{ }^{32} \mathrm{~S}\right] /\left[{ }^{34} \mathrm{~S}\right]=27 \pm 3$ observed by Jewitt et al. (1997) for the CS radical and than the cosmic isotopic ratio $\left[{ }^{32} \mathrm{~S}\right] /\left[{ }^{34} \mathrm{~S}\right]=22.5$ (Anders \& Grevesse 1989).

\section{Results: Radicals and ions}

Many radicals and ions were covered in our survey ${ }^{3}$. Some of them are listed in Table 6:

\section{- SiC (silicon carbide);}

- $\mathrm{SiO}$ (silicon monoxide);

\footnotetext{
${ }^{3}$ Our observations of $\mathrm{CN}, \mathrm{CO}^{+}, \mathrm{HCO}^{+}$and $\mathrm{H}_{3} \mathrm{O}^{+}$are presented and briefly discussed by Lis et al. (1999) and Henry et al. (2001).
} 


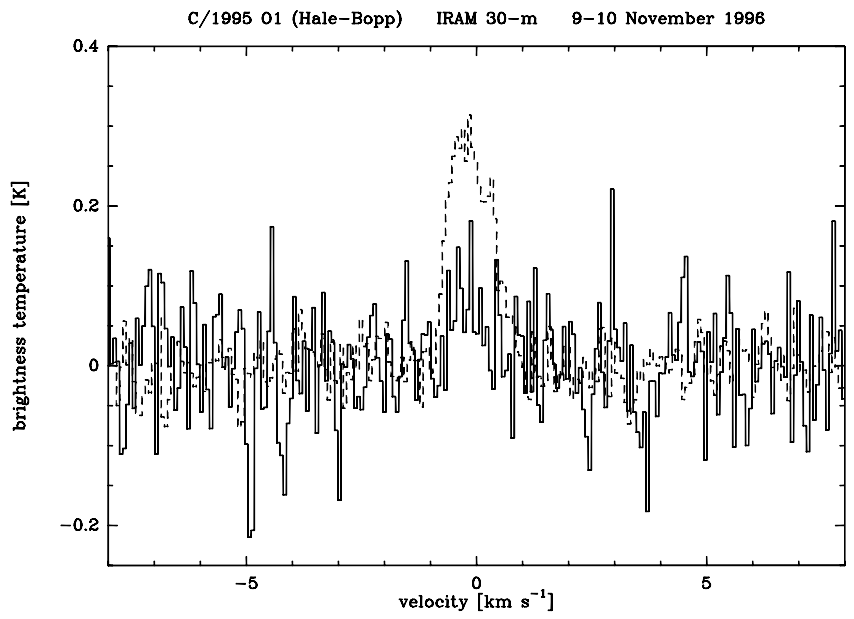

Fig. 4. The $\mathrm{H}_{2}^{34} \mathrm{~S}$ line observed at IRAM in comet Hale-Bopp (average of 9 and 10 November 1996). The $\mathrm{H}_{2}^{32} \mathrm{~S}$ line observed on 9 November is shown for comparison in dashes, scaled down by a factor of 5 .

- HCO radical;

- HNO radical;

- HCP radical;

- $\mathrm{HOCO}^{+}$(protonated carbon dioxide);

- $\mathrm{H}_{2} \mathrm{COH}^{+}$(protonated formaldehyde);

- $\mathrm{HCNH}^{+}$(protonated hydrogen cyanide);

- $\mathrm{N}_{2} \mathrm{H}^{+}$(protonated dinitrogen).

For completeness, we also mention our search for the $\mathrm{CH}$ radical lines at $9 \mathrm{~cm}$ wavelength performed in May 1997 with the Nançay radio telescope, reported by Crovisier et al. (2002).

These species obviously cannot be parent molecules. We do not provide any evaluation of their upper limits in terms of production rates. Their observations are listed in Table 6 . The special case for the $\mathrm{C}_{2} \mathrm{H}$ radical is discussed in the next subsection.

\subsection{Ethynyl radical $\left(\mathrm{C}_{2} \mathrm{H}\right)$}

$\mathrm{C}_{2} \mathrm{H}$ (ethynyl radical) could be a photo-degradation product of many hydrocarbon species. It could also be responsible for the $2.44 \mu \mathrm{m}$ unidentified features in infrared spectra of comets (S.J. Kim, pers. comm.). Altwegg et al. (1999) suggested the possible presence of $\mathrm{C}_{2} \mathrm{H}$ in $1 \mathrm{P} /$ Halley coma, from mass spectroscopy, with $Q\left[\mathrm{C}_{2} \mathrm{H}\right] / Q\left[\mathrm{H}_{2} \mathrm{O}\right]=1-6 \times 10^{-4}$.

Marginal features are present at 262.005 and $262.066 \mathrm{GHz}$ in a CSO spectrum, which also shows features of SO $\left(6_{6}-5_{6}\right)$, $\mathrm{H}^{13} \mathrm{CN} \mathrm{J(3-2)}$ and methanol (Fig. 5). They might be due to the $N(3-2) J(7 / 2-5 / 2)$ and $J(5 / 2-3 / 2)$ transitions of this radical. Both features are broader $\left(\Delta v \approx 15 \mathrm{~km} \mathrm{~s}^{-1}\right)$ than other cometary lines. This could be explained by: (i) the hyperfine structure of these multiplet transitions; (ii) the kinematics of $\mathrm{C}_{2} \mathrm{H}$; coming from multi-step photodissociation of hydrocarbons, $\mathrm{CH}_{2}$ could have higher velocities than parent molecules, when produced with an ejection velocity outside the inner collisional region. The feature corresponding to the $N(3-2) J(7 / 2-5 / 2)$ transition is not centred exactly on the expected frequency, however.

We have evaluated the expected signal of $\mathrm{C}_{2} \mathrm{H}$ expected from the photodissociation of acetylene. We assumed

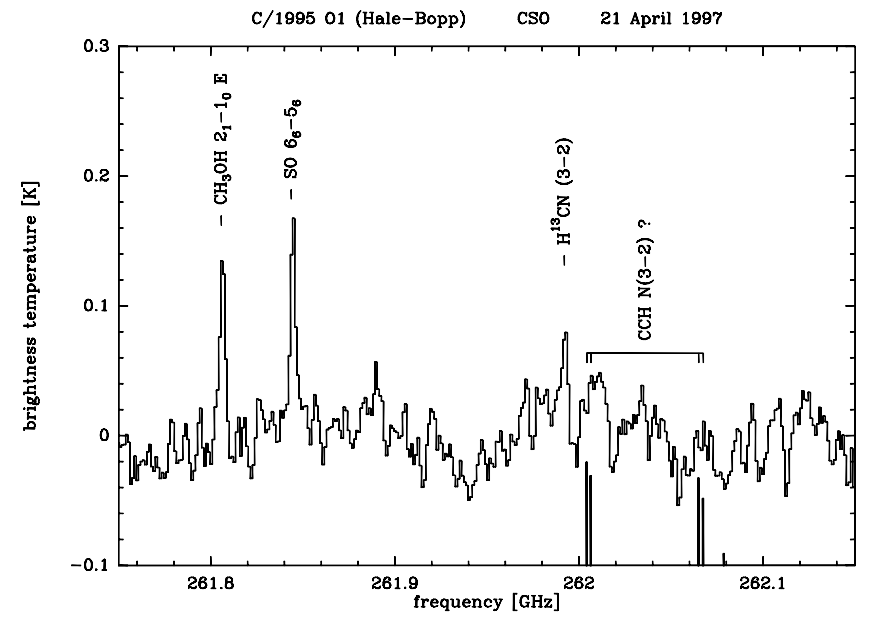

Fig. 5. The $\mathrm{C}_{2} \mathrm{H}$ lines tentatively observed at the CSO in comet HaleBopp. Bars indicate the relative intensities of the $\mathrm{C}_{2} \mathrm{H}$ multiplet. Lines of $\mathrm{CH}_{3} \mathrm{OH}$, SO and $\mathrm{H}^{13} \mathrm{CN}$ (in the lower sideband) were observed simultaneously.

$Q\left[\mathrm{C}_{2} \mathrm{H}_{2}\right]=0.3 \%$ that of water (Dello Russo et al. 2001). $\mathrm{C}_{2} \mathrm{H}_{2}$ photodissociates preferentially in $\mathrm{C}_{2} \mathrm{H}$ with a rate of $10^{-5} \mathrm{~s}^{-1}$ at $1 \mathrm{AU}$ (Huebner et al. 1992; Boyé et al. 2002). We assumed that the scale length of $\mathrm{C}_{2} \mathrm{H}$ (whose lifetime is unknown) is larger than the instrumental field of view. We derive $\int T_{B} \mathrm{~d} v=150$ and $110 \mathrm{mK} \mathrm{km} \mathrm{s}{ }^{-1}$ for the $N(3-2) J(7 / 2-5 / 2)$ and $J(5 / 2-3 / 2)$ blends, respectively. The corresponding features in Fig. 5 are 600 and $200 \mathrm{mK} \mathrm{km} \mathrm{s}^{-1}$, respectively. This is a factor two to four larger than expected from the model. However, we consider that the identification is plausible, considering model uncertainties and the expected contributions of $\mathrm{C}_{2} \mathrm{H}_{6}$ and of still undetected other hydrocarbons to the production of the $\mathrm{C}_{2} \mathrm{H}$ radical.

\section{Discussion and conclusion}

\subsection{Methodological issues}

From the spectral survey of comet Hale-Bopp at radio wavelengths, the detection of more than 20 molecules, molecular ions, radicals and isotopologues was achieved. The present analysis results in setting upper limits on the abundances of $\approx 25$ additional molecules and 5 isotopologues (Table 7). The present survey is a significant improvement over those made previously at IRAM on comets C/1989 X1 (Austin) and C/1990 K1 (Levy) (Crovisier et al. 1993), because comet Hale-Bopp had a much higher gas production rate and because of the higher spectral coverage, thanks to the CSO. The improvement over the CSO survey made on comet C/1996 B2 (Hyakutake) (Lis et al. 1997) is not so important in terms of sensitivity (because the close approach of this comet to Earth compensated its more modest gas production rate), but the present survey is more comprehensive because longer observing time could be scheduled.

Spectral confusion is still not a problem (in contrast with galactic sources such as Orion or the Galactic Centre): cometary lines are narrow, and rotational temperatures are low. One should remark, however, that in a bright comet such 


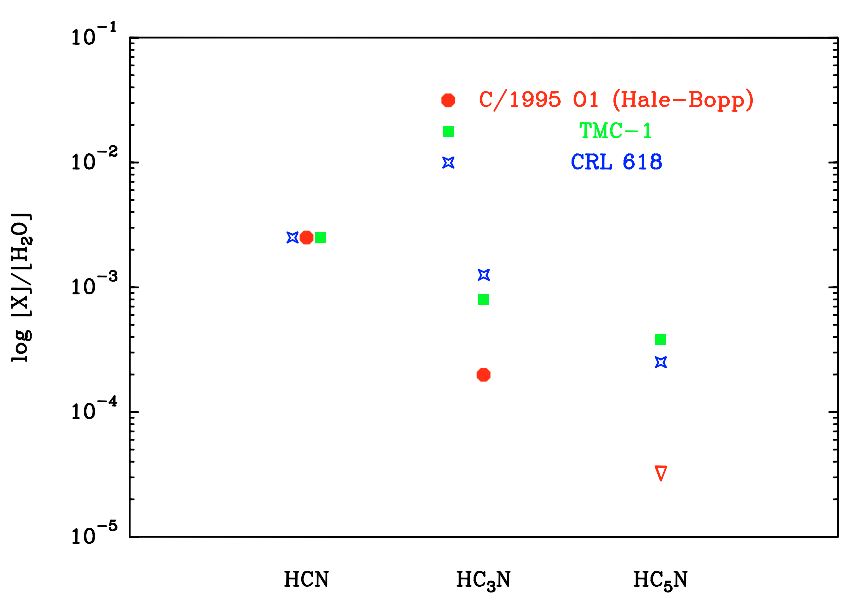

Fig. 6. The relative abundances of linear cyanopolyynes in comet Hale-Bopp (Bockelée-Morvan et al. 2000 and present work), in the dark cloud TMC-1 (Irvine et al. 1987) and in the proto planetary nebula CRL 618 (Cernicharo et al. 2001). For the comet, the abundances are plotted relative to water and the triangle indicates an upper limit. For the interstellar and circumstellar sources, the abundances are normalized to that of $\mathrm{HCN}$.

as comet Hale-Bopp, the advantage of the large gas production rate, yielding more intense lines, is in part counterbalanced by the larger expansion velocity and the higher rotational temperature.

The detections are becoming harder when molecular species are getting more complex and heavier. This is easily explained not only because these species are less abundant, but also because their emission is distributed over much more lines (partition function effect), at longer wavelengths. This can be seen from Table 7: very low upper limits could be set to linear species such as $\mathrm{NaCl}$ or $\mathrm{HC}_{5} \mathrm{~N}$, whereas limits on species such as glycine or dimethyl ether are not stringent.

What is the reliability of our upper limits (Table 7)? To convert column densities into production rates, a parent molecule spatial distribution has been assumed. For secondary species (resulting from chemical processing) or species coming from a distributed source such as organic grains, the listed upper limits are underestimated. For many species, photodissociation rates are lacking (see Table 3 ). In this case, the default value which is assumed $\left(10^{-4} \mathrm{~s}^{-1}\right)$ corresponds, for the average observing conditions, to a scalelength equal to the instrumental field-of-view radius at a frequency of $135 \mathrm{GHz}$ for the IRAM 30-m, of $270 \mathrm{GHz}$ for the IRAM PdBI, and of $400 \mathrm{GHz}$ for the CSO. It is thus a crucial parameter. As a consequence, upper limits for short-lived species (which therefore "do not fill the beam") may be significantly underestimated.

\subsection{Consequences for the chemistry of comets}

The present work completes the inventory of molecules in comet Hale-Bopp presented by Bockelée-Morvan et al. (2000). Molecular abundances are rapidly decreasing with increasing complexity. This is clear for the homologous series of linear cyanopolyynes (Fig. 6). This can also be seen from the limit

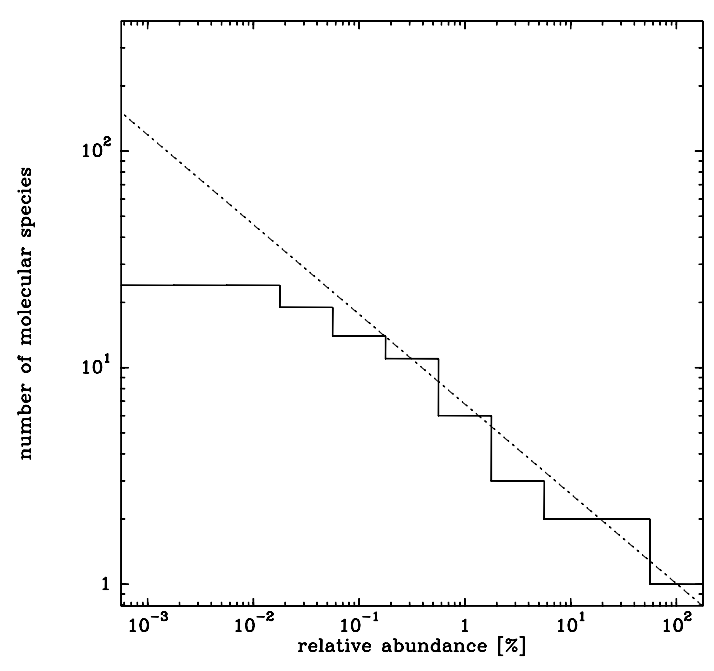

Fig. 7. The number of detected molecular parent species with production rates (relative to water in percent) larger than a given abundance value. The dashed line is a guess of the true distribution.

[ethanol] $/[$ methanol] $<1 / 25$. The limit [acetic acid]/[formic acid] $<2 / 3$ is not so stringent.

This decrease of abundance with increasing complexity is not observed for hydrocarbons: abundances for $\mathrm{C}_{2} \mathrm{H}_{2}$ and $\mathrm{C}_{2} \mathrm{H}_{6}$ are not much less than that of $\mathrm{CH}_{4}$ (Bockelée-Morvan et al. 2004 and references therein). This might be explained by drastic fractionation following condensation/sublimation of this hypervolatile species (Notesco et al. 1997).

\subsection{Future prospects}

Which molecular species are still to be discovered in comets? There are certainly many more molecules to be detected at the $10^{-4}-10^{-5}$ abundance level, but we may wonder if we are missing important molecules at the $10^{-3}$ or more level. Figure 7 shows the cumulative histogram of the number $N$ of detected species as a function of their abundance $X$. We have also drawn the power law $N \propto X^{-0.4}$, suggested by the data from the most abundant species. If realistic, this law would imply that observational biases affect species with abundances $<10^{-3}$, and that we should expect $\approx 100$ species with $X>10^{-5}$.

The presence of unidentified features in cometary spectra already points to the existence of further species. In the visible, many features are noted (e.g., Brown et al. 1996; Cochran \& Cochran 2002), presumably due to radicals. Others were recently revealed in the infrared with the Keck/NIRSPEC instrument (Mumma et al. 2001a,b), that could be due to parent molecules. Several unidentified lines at the 3 to $5-\sigma$ level of confidence are also noted in our radio survey. Some of them are listed in Table 9.

In the radio domain, future progress will depend upon the apparition of bright, unexpected comets, since there is no short-period comet with high production rate (i.e., $Q\left[\mathrm{H}_{2} \mathrm{O}\right]>$ $10^{29} \mathrm{~s}^{-1}$ ) expected in the next years. The emergence of new instruments in the next decade, such as the Atacama Large Millimetre Array, which will provide an increase of sensitivity, or the Herschel Space Observatory, which will open a new 
Table 9. Some unidentified lines observed at the IRAM 30-m telescope.

\begin{tabular}{rlr}
\hline \hline $\begin{array}{r}\text { Frequency } \\
{[\mathrm{MHz}]}\end{array}$ & $\begin{array}{l}\text { Date } \\
\text { dd/mm/yyyy }\end{array}$ & $\begin{array}{r}\int T_{b} \mathrm{~d} v \\
{\left[\mathrm{mK} \mathrm{km} \mathrm{s}^{-1}\right]}\end{array}$ \\
\hline 96732.7 & $29 / 05 / 1997$ & $42 \pm 16$ \\
105468.6 & $03 / 04 / 1997$ & $65 \pm 21$ \\
105551.9 & $03 / 04 / 1997$ & $129 \pm 34$ \\
106494.8 & $03 / 04 / 1997$ & $54 \pm 17$ \\
106911.5 & $03 / 04 / 1997$ & $62 \pm 16$ \\
140782.3 & $03 / 04 / 1997$ & $180 \pm 39$ \\
140792.5 & $03 / 04 / 1997$ & $174 \pm 39$ \\
140794.1 & $03 / 04 / 1997$ & $125 \pm 39$ \\
146964.3 & $03 / 04 / 1997$ & $75 \pm 36$ \\
147094.4 & $03 / 04 / 1997$ & $125 \pm 31$ \\
157014.5 & $03 / 04 / 1997$ & $244 \pm 74$ \\
203403.0 & $08 / 04 / 1997$ & $203 \pm 50$ \\
218465.0 & $03 / 04 / 1997$ & $290 \pm 60$ \\
226777.9 & $11 / 04 / 1997$ & $245 \pm 73$ \\
227677.6 & $05 / 04 / 1997$ & $137 \pm 38$ \\
236040.5 & $04 / 04 / 1997$ & $358 \pm 68$ \\
\hline
\end{tabular}

Some of these features do coincide with molecular lines, but the identifications have been ruled out because the transitions occur between high energy levels, or the same species is expected to have stronger lines that are not seen.

spectral range in the submillimetric domain, will certainly contribute new discoveries.

The present work clearly shows the limits of chemical analysis of comets by remote sensing. More complex species such as amino acids will have to be investigated by space probes with in situ techniques such as gas chromatography, or will need the return to Earth of cometary nucleus samples.

Acknowledgements. We are grateful to all the persons who participated in these target-of-opportunity observations: R. Bachiller, D. J. Benford, J. K. Davies, E. Gérard, B. Germain, M. Houde, D. Mehringer, R. Moreno, G. Paubert, T. G. Phillips, H. Rauer, \& J. E. Wink. We thank L. H. Coudert, J. Demaison, E. Herbst, I. Kleiner \& G. Wlodarczak who contributed to this work by providing lists of molecular transitions. The JPL and Cologne databases were crucial for the preparation of the observations, quick-look reduction and final analysis. This research has been supported by the Centre national de la recherche scientifique, the Programme national de planétologie de l'Institut national des sciences de l'univers, and NSF grant AST 2209008 to the Caltech Submillimeter Observatory. IRAM is supported by INSU/CNRS (France), MPG (Germany) and IGN (Spain).

\section{References}

Altwegg, K., Balsiger, H., \& Geiss, J. 1999, Space Sci. Rev., 90, 3 Altwegg, K., \& Bockelée-Morvan, D. 2003, Space Sci. Rev., 106, 139 Anders, R., \& Grevesse, E. 1998, Geochim. Cosmochim. Acta, 53, 197

Anderson, T., Crownover, R., Herbst, E., \& de Lucia, F. C. 1988, ApJS, 67, 135
Bettens, F. L., Sastry, K. V. L. N., Herbst, E., et al. 1999, ApJ, 510, 789

Bird, M. K., Janardhan, P., Wilson, T. L., et al. 1999, Earth Moon Planets, 78, 21

Biver, N., Bockelée-Morvan, D., Colom, P., et al. 1999, Earth Moon Planets, 78, 5

Biver, N., Bockelée-Morvan, D., Crovisier, J., et al. 2002, Earth Moon Planets, 90, 323

Bockelée-Morvan, D., Crovisier, J., Mumma, M. J., \& Weaver, H. A. 2004, in Comets II, ed. M. Festou, H. U. Keller, \& H. A. Weaver (Tucson: Univ. Arizona Press), in press

Bockelée-Morvan, D., Gautier, D., Lis, D. C., et al. 1998, Icarus, 133, 147

Bockelée-Morvan, D., Lis, D. C., Wink, J., et al. 2000, A\&A, 353, 1101

Bockelée-Morvan, D., Wink, J., Despois, D., et al. 1999, Earth Moon Planets, 78, 67

Boyé, S., Campos, A., Douin, S., et al. 2002, J. Chem. Phys., 115, 3566

Brown, M. E., Bouchez, A. H., Spinrad, A. H., \& Johns-Krull, C. M. 1996, AJ, 112, 1197

Canaves, M. V., De Almeida, A. A., Boice, D. C., \& Sanzovo, G. C. 2002, Earth Moon Planets, 90, 335

Ceccarelli, C., Loinard, L., Castet, A., Faure, A., \& Lefloch, B. 2000, A\&A, 362, 1122

Cernicharo, J., Heras, A. M., Tielens, A. G. G. M., et al. 2001. ApJ, 546, L123

Charnley, S. B., Rodgers, S. D., \& Ehrenfreund, P. 2001, A\&A, 378, 1024

Charnley, S. B., Tielens, A. G. G. M., \& Rogers, S. D., 1997, ApJ, 482, L203

Cochran, A. L., \& Cochran, W. D. 2002, Icarus, 157, 297

Colom, P., Crovisier, J., Bockelée-Morvan, D., Despois, D., \& Paubert, G. 1992, A\&A, 264, 270

Colom, P., Gérard, E., Crovisier, J., et al. 1999, Earth Moon Planets, 78,37

Combes, F., Nguyen-Q-Rieu, \& Wlodarczak, G. 1996, A\&A, 308, 618

Combi, M. R., Reinard, A. A., Bertaux, J.-L., Quemarais, E., \& Mäkinen, T. 2000, Icarus, 144, 191

Coudert, L. H., \& Hougen, J. T. 1990, J. Mol. Spect., 139, 259

Cremonese, G., Boehnhardt, H., Crovisier, J., et al. 1997, ApJ, 490, L199

Cremonese, G., Huebner, W. F., Rauer, H., \& Boice, D. C. 2002, Adv. Space Res., 29, 1187

Crifo, J.-F., \& Slanina, Z. 1991, ApJ, 383, 351

Cronin, J. R., \& Chang, S. 1993, in The Chemistry of Life's Origin, ed. J. M. Greenberg, C. X. Mendoza-Gomez, \& V. Pirronello (Dordrecht: Kluwer), 209

Crovisier, J. 1994, J. Geophys. Res., 99(E2), 3777

Crovisier, J. 1998, Faraday Discuss., 109, 437

Crovisier, J., Bockelée-Morvan, D., Biver, N., et al. 2004, A\&A, 418, L35

Crovisier, J., Bockelée-Morvan, D., Colom, P., Despois, D., \& Paubert, G. 1993, A\&A, 269, 527

Crovisier, J., Colom, P., Gérard, E., Bockelée-Morvan, D., \& Bourgois, G. 2002, A\&A, 393, 1053

Dello Russo, N., Mumma, M. J., DiSanti, M. J., Magee-Sauer, K., \& Novak, R. 2001, Icarus, 155, 163

Despois, D. 1999, Earth Moon Planets, 79,103

Eberhardt, P., Meier, R., Krankowsky, D., \& Hodges, R. R. 1994, A\&A, 288, 315

Ehrenfreund, P., Berstein, M. P., Dworkin, J. P., Sandford, S. A., \& Allamandola, L. J. 2001a, AJ, 550, L95 
Ehrenfreund, P., Glavin, D. P., Botta, O., Cooper, G., \& Bada, J. L. 2001b, Proc. Nat. Acad. Scie., 98, 2138

Grevesse, N., \& Sauval, A. J. 1998, Space Scie. Rev., 85, 161

Groner, P., Albert, S., Herbst, E., \& de Lucia, F. C. 1998, ApJ, 500, 1059

Grün, E., Hanner, M. S., Peschke, S. B., et al. 2001, A\&A, 377, 1098

Hatchell, J., Roberts, H., \& Millar, T. J. 1999, A\&A, 346, 227

Helbert, J., Rauer, H., Boice, D., \& Huebner, W. 2000, BAAS, 32, 1071

Helminger, P., De Lucia, F. C., \& Kirchoff, W. H. 1973, J. Phys. Chem. Ref. Data, 2, 215

Henry, F., Crovisier, J., Bockelée-Morvan, D., Rauer, H., \& Lis, D. 2001, Astrophys. Space Scie., 277, 303

Hersant, F., Gautier, D., \& Huré, J.-M. 2001, ApJ, 554, 391

Hollis, J. M., Pedelty, J. A., Snyder, L. E., et al. 2003, ApJ, 588, 353

Huebner, W. F., Keady, J. J., \& Lyon, S. P. 1992, Astrophys. Space Scie., 195, 1

Ilyushin, V. V., Alekseev, E. A., Dyubko, S. F., et al. 2001, J. Mol. Spect., 205, 286

Ilyushin, V. V., Alekseev, E. A., Dyubko, S. F., \& Kleiner, I. 2003, J. Mol. Spect., 220, 170

Irvine, W. M., Dickens, J. E., Lovell, A. J., et al. 1998, Faraday Discus., 109, 475

Irvine, W. M., Dickens, J. E., Lovell, A. J., et al. 1999, Earth Moon Planets, 78, 29

Irvine, W. M., Goldsmith, P. F., \& Hjalmarson, Å. 1987, in Interstellar Processes, ed. D. J. Hollenbach, \& Jr H. A. Thronson (Reidel Publishing Company), 561

Irvine, W. M., Schloerb, F. P., Crovisier, J., Fegley, B., \& Mumma, M. J. 2000a, in Protostars and Planets IV, ed. V. Mannings, A. Boss, \& S. Russel (Tucson: Univ. of Arizona Press), 1159

Irvine, W. M., Senay, M., Lovell, A. J., et al. 2000b, Icarus, 143, 412

Jacq, T., Walmsley, C. M., Mauersberger, R., et al. 1993, A\&A, 271, 276

Jewitt, D. C., Matthews, H. E., Owen, T., \& Meier, R. 1997, Science, 278, 90

Kleiner, I., Lovas, F. J., \& Godefroid, M. 1996, J. Phys. Chem. Ref. Data, 25, 1113

Krasnopolsky, V. A. 1991, A\&A, 245, 310

Kuan, Y.-J., Charnely, S. B., Hueng, H.-C., Tseng, W.-L., \& Kisiel, Z. 2003, ApJ, 593, 848

Lis, D. C., Keene, J., Young, T. G., et al. 1997, Icarus, 130, 355

Lis, D. C., Mehringer, D., Benford, D., et al. 1999, Earth Moon Planets, 78, 13

Lovas, F. J., Kawashima, Y., Grabow, J.-U., et al. 1995, ApJ, 455, L201
Meier, R., \& Owen, T. C. 1999, Space. Scie. Rev., 90, 33

Meier, R., Owen, T. C., Jewitt, D. C., et al. 1998b, Science, 279, 1707

Meier, R., Owen, T. C., Matthews, H. E., et al. 1998a, Science, 279, 842

Miao, Y., Snyder, L. E., Kuan, Y.-J., \& Lovas, F. J. 1994, BAAS, 26, 906

Millar, T. J. 2000, in Science with the Atacama Large Millimeter Array, ed. A. Wootten, ASP Conf. Ser., 235, 45

Müller, H. S. P., Thorwirth, S., Roth, D. A., \& Winnewisser, G. 2001, A\&A, 370, L49 (electronic files available from http://www.cdms.de)

Mumma, M. J., Dello Russo, N., DiSanti, M. A., et al. 2001a, Science, 292,1334

Mumma, M. J., McLean, I. S., DiSanti, M. A., et al. 2001b, ApJ, 546, 1183

Notesco, G., Laufer, D., \& Bar-Nun, A. 1997, Icarus, 125, 471

Nummelin, A., Dickens, J. E., Bergman, P., et al. 1998, A\&A, 337, 275

Parise, B., Ceccarelli, C., Tielens, A. G. G. M., et al. 2002, A\&A, 393, L49

Pearson, J. C., Sastry, K. V. L. N., Herbst, E., \& de Lucia, F. C. 1997, ApJ, 480, 420

Pickett, H. M., Poynter, R. L., Cohen, E. A., et al. 1998, J. Quant. Spect. Rad. Trans., 60, 883 (electronic files available from http://spec.jpl.nasa.gov)

Rodgers, S. D., \& Charnley, S. B. 2001, MNRAS, 323, 84

Saito, S., Ozeki, H., Ohishi, M., \& Yamamoto, T. 2000, ApJ, 535, 227

Scherer, M., Havenith, M., Mauersberger, R., \& Wilson, T. L. 1998, A\&A, 335, 1070

Schleicher, D. G., Lederer, S. M., Millis, R. M., \& Farnham, T. L. 1997, Science, 275, 1913

Snyder, L. E. 1997, Origins Life Evol. Biosphere, 27, 115

Weiler, M., Rauer, H., Knollenberg, J., Jorda, L., \& Helbert, J. 2003, A\&A, 403, 313

Wink, J., Bockelée-Morvan, D., Despois, D., et al. 1999, Earth Moon Planets, 78, 63

Winnewisser, M., \& Winnewisser, B. P. 1971, Z. Naturforsch., 26a, 128

Wlodarczak, G., \& Demaison, J. 1988, A\&A, 192, 313

Woodney, L. M. 2000. Ph.D. Thesis, Univ. of Maryland

Woodney, L. M., A'Hearn, M. F., Lisse, C., \& McMullin, J. 1999, Earth Moon Planets, 78, 69

Zwart, E., ter Meulen, J. J., Meerts, W. L., \& Coudert, L. H. 1991, J. Mol. Spect., 147, 27 